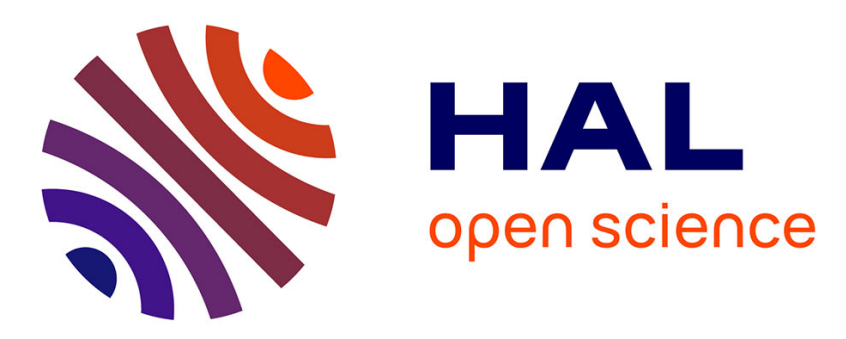

\title{
Two-flux and multiflux matrix models for colored surfaces
}

Mathieu Hébert, Patrick Emmel

\section{To cite this version:}

Mathieu Hébert, Patrick Emmel. Two-flux and multiflux matrix models for colored surfaces. M. Kriss. Handbook of Digital Imaging, 2, Wiley, pp.1234-1278, 2015, 978-0-470-51059-9. hal-01179591

\section{HAL Id: hal-01179591 \\ https://hal.science/hal-01179591}

Submitted on 23 Jul 2015

HAL is a multi-disciplinary open access archive for the deposit and dissemination of scientific research documents, whether they are published or not. The documents may come from teaching and research institutions in France or abroad, or from public or private research centers.
L'archive ouverte pluridisciplinaire HAL, est destinée au dépôt et à la diffusion de documents scientifiques de niveau recherche, publiés ou non, émanant des établissements d'enseignement et de recherche français ou étrangers, des laboratoires publics ou privés. 


\section{Two-flux and multiflux matrix models for colored surfaces}

CHAPTER · FEBRUARY 2015

CITATION

1

2 AUTHORS:
VIEWS

20 


\title{
Two-flux and multiflux matrix models for colored surfaces
}

\author{
Mathieu Hébert \\ Université de Lyon, Université Jean Monnet de Saint-Etienne, CNRS UMR 5516 Laboratoire \\ Hubert Curien, F-42000, Saint-Etienne, France. \\ and \\ Patrick Emmel \\ 14 rue de Münchendorf, 68220 Folgensbourg, France.
}

\begin{abstract}
This paper presents various extensions of the so-called two-flux models for prediction of reflectance and transmittance of diffusing media, i.e. the Kubelka-Munk model, introduced in [HDI062], and the extension of Kubelka-Munk for stacks of diffusing layers. A first matrix formulation of the Kubelka-Munk differential equations leads to a matrix framework based on transfer matrices, which can be extended to stacks of diffusing layers, stacks of nonscattering films, and stacks of scattering and non-scatterings films as a generalization of the WilliamsClapper model for prediction of the reflectance of paper photographs, each of these configurations being illustrated through various examples. This paper also exposes the limits of the two flux approach and shows that the matrix formalism extends in a straightforward manner to multiflux models, where the size of the matrices is increased.
\end{abstract}

\section{INTRODUCTION}

Although prints and paints are often considered as "surfaces", they are actually made of various layers of more or less scattering and absorbing media. This is obvious in printing where a strongly scattering substrate (e.g. paper) is coated with absorbing, almost nonscattering substances (inks). In glaze painting, the board is first coated with a white diffusing paint layer aimed at reflecting light in a uniform way in every direction; it is then coated with several absorbing layers, almost nonscattering, for coloration; it is finally coated with a clear varnish for protection [1,2]. More complex structures may be found everywhere, for example photos under glass or books covered by a protection film: light is reflected multiple times between the colored object and the protecting element, thus changing the color rendering of 
the object. Optical models predicting the color rendering of transparent films, glass plates, paints or printed papers taken individually are relatively well known (see [HDI062], Section 4.2, and [HDI051]). However, the combination of these models, necessary when various elements are on top of each other, is less common. In many cases, i.e. when each of the stacked elements is strongly scattering or non-scattering, the spectral reflectance or transmittance of the object can be predicted using a two-flux model describing the forward and backward propagation of fluxes and their mutual exchanges due to scattering in layers and reflections by their interfaces. The two-flux approach is theoretically permitted when the angular distribution of light does not vary after several reflections between the stacked elements [3]: this is the case for strongly scattering layers illuminated by a Lambertian flux, addressed in Sections 2 and 3, and for nonscattering layers illuminated by a collimated flux, addressed in Section 4. The two-flux approach also enables addressing stacks where strongly scattering layers and non-scattering layers are alternated (Section 5).

In its classical form introduced by Kubelka and Munk [4, 5], the two-flux model is expressed in terms of flux attenuations due to scattering and absorption, parameterized by scattering and absorption coefficients. The flux attenuations in infinitesimal sublayers are written under the form of scalar differential equations, or equivalently under the form of one vector differential equation, involving an attenuation matrix, presented in Section 2. By integrating this vector differential equation, the incoming and outgoing fluxes at both sides are related through one matrix, which models the flux transfers between each other and is thus qualified as transfer matrix. This transfer matrix formalism is shown to be very convenient when different media are coated upon each other, or when several slides of media are stacked to each other. As Kubelka in his paper of 1954 dedicated to non-homogeneous layers [6], the matrix model can be extended to reflecting-transmitting components with distinct reflectances on their two sides. The transferred flux fractions are thus represented by the reflectances and transmittances of the layers, and possibly of their interfaces, which can be either predicted or deduced from spectral measurements. Layers may have any thickness (provided they are thicker than the coherence length of the incident light) and may be themselves composed of various elements. Several examples will be developed in Section 3 to 5, illustrating the easy computation of the reflectance and transmittance of stacked elements knowing, by measurement or by prediction, their individual reflectances and transmittances. The matrix formalism has also the advantage to be easily extensible to multiflux when several directions of light need to be treated in parallel, as shown in Section 6 in the case of homogenous layers. 


\section{REVISITING THE KUBELKA-MUNK MODEL (TWO-FLUX MODEL)}

This section revisits the Kubelka-Munk model whose classical formulation has been introduced in [HDI062], Section 7. We present here a matrix formulation of the differential equations which simplifies the computation of reflectance and transmittance of homogenous scattering layers and enables appreciable extensions.

\subsection{Matrix method to solve the Kubelka-Munk differential equation system}

The Kubelka-Munk model considers a thin slice of diffusing medium whose thickness $d z$ is small compared to its two other dimensions. Let us denote as $j$ a diffuse light flux oriented upwards (increasing altitude $z$ ) and as $i$ a diffuse light flux oriented downwards (decreasing altitude $z$, see Figure 1). We assume that all variations of the light fluxes are only due to absorption and scattering. We denote as $K$ the linear absorption coefficient and as $S$ the linear backscattering coefficient. Flux $i$ and $j$, as well as coefficients $K$ and $S$ may be functions of the wavelength. In order to simplify the notations, we omit their dependence on wavelength and implicitly assume that they may have distinct values in the different wavebands of the visible spectrum. This also holds for every equation in this paper.

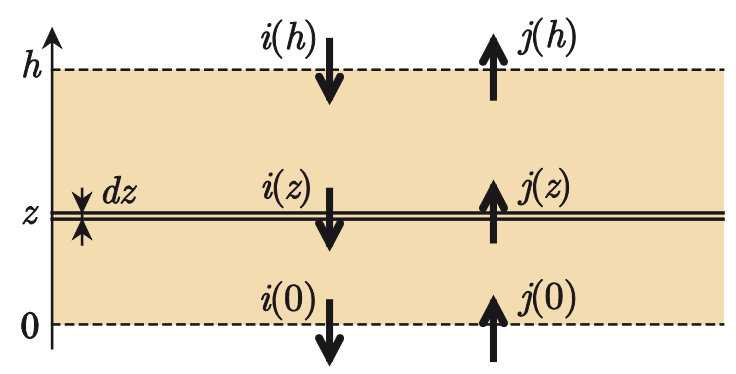

Figure 1: Upwards and downwards fluxes in a layer of thickness $h$.

While crossing the slice of medium, flux $j$ decreases by an amount $K \cdot j \cdot d z$ due to absorption and also by an amount $S \cdot j \cdot d z$ due to back-scattering. It increases by the amount of light $S \cdot i \cdot d z$ lost by back-scattering if the flux $i$ while crossing the same slice of medium in the opposite direction:

$$
d j=-(K+S) \cdot j \cdot d z+S \cdot i \cdot d z
$$

Analyzing flux $i$ leads to a similar equation. Since the orientation of this flux is opposite, negative signs are introduced:

$$
d i=S \cdot j \cdot(-d z)-(K+S) \cdot i \cdot(-d z)=-S \cdot j \cdot d z+(K+S) \cdot i \cdot d z
$$


Note that the Kubelka-Munk model does not distinguish between forward scattered light and light that wasn't deviated. In other words, this model makes no distinction between a transparent medium, i.e. an absorbing but not scattering medium, and a hazy medium where scattered light reduces the contrast of objects viewed through it. Haze is the percentage of transmitted light that is scattered so that its direction deviates more than an angle of $2.5^{\circ}$ $(0.044 \mathrm{rad})$ from the direction of the incident beam [7].

Combining equations (1) and (2) yields a system of differential equations:

$$
\left\{\begin{array}{l}
\frac{d j}{d z}=-(K+S) j+S i \\
\frac{d i}{d z}=-S j+(K+S) i
\end{array}\right.
$$

There are several ways to solve this system of equations. This chapter will focus on a matrix based approach that enables addressing more advanced models. Let us look at the system of equations (3) as a differential equation in a vector space which can be written:

$$
\frac{d}{d z}\left(\begin{array}{l}
j \\
i
\end{array}\right)=\mathbf{M}_{K, S} \cdot\left(\begin{array}{l}
j \\
i
\end{array}\right)
$$

where $\mathbf{M}_{K, S}$ denotes the matrix of Kubelka-Munk coefficients, or "attenuation matrix":

$$
\mathbf{M}_{K, S}=\left(\begin{array}{cc}
-(K+S) & S \\
-S & (K+S)
\end{array}\right)
$$

Before solving equation (4), let us first recall that the solution for a scalar differential equation of the first order of the form

$$
\frac{d u}{d z}=m \cdot u
$$

is

$$
u(z)=e^{m\left(z-z_{0}\right)} u\left(z_{0}\right)
$$

Similarly, the solution for the vector differential equation (4) is

$$
\left(\begin{array}{c}
j(z) \\
i(z)
\end{array}\right)=\exp \left[\left(\begin{array}{cc}
-(K+S) & S \\
-S & (K+S)
\end{array}\right)\left(z-z_{0}\right)\right] \cdot\left(\begin{array}{c}
j\left(z_{0}\right) \\
i\left(z_{0}\right)
\end{array}\right)
$$

where 'exp' here denotes the matrix exponential function defined by the series:

$$
\exp (\mathbf{M})=\sum_{i=0}^{\infty} \frac{\mathbf{M}^{i}}{i !}=\mathbf{I}+\frac{\mathbf{M}}{1 !}+\frac{\mathbf{M}^{2}}{2 !}+\frac{\mathbf{M}^{3}}{3 !}+\ldots
$$


where I denotes the $2 \times 2$ identity matrix.

Thus, by integrating the vector differential equation (4) between $z_{0}=0$ and $z=h$, one obtains the following equation relating fluxes $i$ and $j$ at the altitudes 0 and $h$ :

$$
\left(\begin{array}{c}
j(h) \\
i(h)
\end{array}\right)=\mathbf{M}_{K, S, h} \cdot\left(\begin{array}{c}
j(0) \\
i(0)
\end{array}\right)
$$

with

$$
\mathbf{M}_{K, S, h}=\exp \left[\mathbf{M}_{K, S} \cdot(h-0)\right]
$$

Note that the matrix $\mathbf{M}_{K, S}$, expressed in terms of the scattering and the absorption coefficients, has a physical dimension in $m^{-1}$, whereas $\mathbf{M}_{K, S, h}$ is dimensionless. This matrix relates the incoming and outgoing fluxes in the layer and models the flux transfers from each other. It can thus be qualified as "transfer matrix", a concept that will be generalized in Section 3.

The exponential of a square matrix always exists and satisfies several interesting properties [8]:

- The exponential of the zero matrix $\mathbf{O}$ is the identity matrix:

$$
\exp (\mathbf{O})=\mathbf{I}
$$

- Let $\mathbf{M}=\mathbf{A} \cdot \mathbf{B} \cdot \mathbf{A}^{-1}$, then

$$
\exp (\mathbf{M})=\exp \left(\mathbf{A} \cdot \mathbf{B} \cdot \mathbf{A}^{-1}\right)=\mathbf{A} \cdot \exp (\mathbf{B}) \cdot \mathbf{A}^{-1}
$$

- Let $\mathbf{A}$ and $\mathbf{B}$ be two matrices that commute with each other, i.e. $\mathbf{A} \cdot \mathbf{B}=\mathbf{B} \cdot \mathbf{A}$, then the exponential of the sum equals the product of the exponentials:

$$
\exp (\mathbf{A}+\mathbf{B})=\exp (\mathbf{A}) \cdot \exp (\mathbf{B})=\exp (\mathbf{B}) \cdot \exp (\mathbf{A})
$$

- The inverse of $\exp (\mathbf{M})$ is $\exp (-\mathbf{M})$.

$$
\exp (\mathbf{M}) \cdot[\exp (\mathbf{M})]^{-1}=\exp (\mathbf{M}) \cdot \exp (-\mathbf{M})=\exp (\mathbf{M}-\mathbf{M})=\mathbf{I}
$$

- Let $x$ be an real number, then

$$
\exp (x \cdot \mathbf{M})=[\exp (\mathbf{M})]^{x}
$$

- The exponential of a diagonal matrix is a diagonal matrix whose entries are the exponential of the entries of the matrix (we will use this fundamental property to compute $\mathbf{M}_{K, S, h}$ introduced in equation (8)): 


$$
\exp \left(\operatorname{diag}\left(v_{1}, \ldots, v_{n}\right)\right)=\operatorname{diag}\left(e^{v_{1}}, \ldots, e^{v_{n}}\right)
$$

$\operatorname{det}\left(\mathbf{M}_{K, S}-v \mathbf{I}\right)$ yields the characteristic polynomial of the matrix $\mathbf{M}_{K, S}$ defined by (5):

$$
\operatorname{det}\left(\mathbf{M}_{K, S}-v \mathbf{I}\right)=v^{2}-2 K S v-K^{2}
$$

whose two roots are the two eigenvalues of $\mathbf{M}_{K, S}$ :

$$
v_{1}=-\sqrt{K^{2}+2 K S}
$$

and

$$
v_{2}=\sqrt{K^{2}+2 K S}
$$

Assuming $\mathbf{M}_{K, S}=\mathbf{V} \cdot \mathbf{D} \cdot \mathbf{V}^{-1}$ where $\mathbf{D}=\operatorname{diag}\left(v_{1}, v_{2}\right)$ is a diagonal matrix, and

$$
\mathbf{V}=\left(\begin{array}{cc}
(K+S)+\sqrt{K^{2}+2 K S} & S \\
S & (K+S)+\sqrt{K^{2}+2 K S}
\end{array}\right),
$$

we have:

$$
\begin{aligned}
\mathbf{M}_{K, S, h} & =\exp \left(\mathbf{M}_{K, S} \cdot h\right) \\
& =\mathbf{V} \cdot \exp (\mathbf{D} \cdot h) \cdot \mathbf{V}^{-1} \\
& =\mathbf{V} \cdot\left(\begin{array}{cc}
e^{v_{1} h} & 0 \\
0 & e^{v_{2} h}
\end{array}\right) \cdot \mathbf{V}^{-1}
\end{aligned}
$$

Finally, equation (8) can be written as the following matrix product:

$$
\left(\begin{array}{l}
j(h) \\
i(h)
\end{array}\right)=\mathbf{V} \cdot\left(\begin{array}{cc}
e^{v_{1} h} & 0 \\
0 & e^{v_{2} h}
\end{array}\right) \cdot \mathbf{V}^{-1} \cdot\left(\begin{array}{c}
j(0) \\
i(0)
\end{array}\right)
$$

or equivalently as the following one:

$$
\left(\begin{array}{l}
j(0) \\
i(0)
\end{array}\right)=\mathbf{V} \cdot\left(\begin{array}{cc}
e^{-v_{1} h} & 0 \\
0 & e^{-v_{2} h}
\end{array}\right) \cdot \mathbf{V}^{-1} \cdot\left(\begin{array}{l}
j(h) \\
i(h)
\end{array}\right)
$$

In this framework, the body reflectance $\rho$ of a layer is given by the ratio $\rho=j(h) / i(h)$. The intrinsic transmittance $\tau_{i}$ is the ratio of the downward oriented fluxes $i(0)$ and $i(h)$ in absence of upward incident flux, i.e. $j(0)=0$ :

$$
\tau_{i}=\left(\frac{i(0)}{i(h)}\right)_{j(0)=0}
$$

Therefore the following holds for a free standing medium: 


$$
\left(\begin{array}{c}
\rho i(h) \\
i(h)
\end{array}\right)=\mathbf{V} \cdot\left(\begin{array}{cc}
e^{v_{1} h} & 0 \\
0 & e^{v_{2} h}
\end{array}\right) \cdot \mathbf{V}^{-1} \cdot\left(\begin{array}{c}
0 \\
\tau_{i} i(h)
\end{array}\right)
$$

i.e., by dividing both members of this equation with $i(h)$,

$$
\left(\begin{array}{l}
\rho \\
1
\end{array}\right)=\mathbf{V} \cdot\left(\begin{array}{cc}
e^{v_{1} h} & 0 \\
0 & e^{v_{2} h}
\end{array}\right) \cdot \mathbf{V}^{-1} \cdot\left(\begin{array}{c}
0 \\
\tau_{i}
\end{array}\right)
$$

A substrate of reflectance $\rho_{g}$ in optical contact with the layer of thickness $h$ at the lower side $(z=0)$ would impose a condition on the boundary $j(0)=\rho_{g} i(0)$ :

$$
\left(\begin{array}{c}
\rho i(h) \\
i(h)
\end{array}\right)=\mathbf{V} \cdot\left(\begin{array}{cc}
e^{v_{1} h} & 0 \\
0 & e^{v_{2} h}
\end{array}\right) \cdot \mathbf{V}^{-1} \cdot\left(\begin{array}{c}
\rho_{g} i(0) \\
i(0)
\end{array}\right)
$$

Developing equation (18) leads to the hyperbolic solution of the Kubelka-Munk model, see equation (26).

\subsection{The classic formulae of the Kubelka-Munk theory}

All classic formulae from the literature (e.g. Ref. [9]), can be derived from equation (14). Let us introduce

$$
a=\frac{K+S}{S}
$$

and

$$
b=\sqrt{a^{2}-1}=\frac{1}{S} \sqrt{K^{2}+2 K S}
$$

under the assumption $S \neq 0$. Note that $K$ and $S, a$ and $b$ are functions of wavelength. Since the eigenvalues $v_{1}$ and $v_{2}$ of $\mathbf{M}_{K, S}$, given by equations (11) and (12), can be written $-b S$ and $b S$ respectively, equation (14) can be written:

$$
\left(\begin{array}{l}
j(h) \\
i(h)
\end{array}\right)=\mathbf{V} \cdot\left(\begin{array}{cc}
e^{-b S h} & 0 \\
0 & e^{b S h}
\end{array}\right) \cdot \mathbf{V}^{-1} \cdot\left(\begin{array}{c}
j(0) \\
i(0)
\end{array}\right)
$$

with

$$
\mathbf{V}=\left(\begin{array}{cc}
S(a+b) & S \\
S & S(a+b)
\end{array}\right)=S\left(\begin{array}{cc}
(a+b) & 1 \\
1 & (a+b)
\end{array}\right)
$$

and

$$
\mathbf{V}^{-1}=\frac{1}{S\left[(a+b)^{2}-1\right]}\left(\begin{array}{cc}
(a+b) & -1 \\
-1 & (a+b)
\end{array}\right)
$$


Developing equation (21) yields:

$$
\left(\begin{array}{c}
j(h) \\
i(h)
\end{array}\right)=\frac{1}{2 b}\left(\begin{array}{cc}
(a+b) e^{-b S h}-(a-b) e^{b S h} & e^{b S h}-e^{-b S h} \\
e^{-b S h}-e^{b S h} & (a+b) e^{b S h}-(a-b) e^{-b S h}
\end{array}\right) \cdot\left(\begin{array}{c}
j(0) \\
i(0)
\end{array}\right)
$$

Note that in the particular case of absence of scattering ( $S=0$ ), equation (22) does not apply.

We need to go back to equation (13), which simplifies as follows:

$$
\left(\begin{array}{c}
j(h) \\
i(h)
\end{array}\right)=\left(\begin{array}{cc}
e^{-K h} & 0 \\
0 & e^{K h}
\end{array}\right) \cdot\left(\begin{array}{l}
j(0) \\
i(0)
\end{array}\right)
$$

\subsection{Layer in optical contact with a background}

Many practical cases, for example inks or paints deposited on a substrate, can be seen as layers in optical contact with a background with given reflectance $\rho_{g}$ (Figure 2).

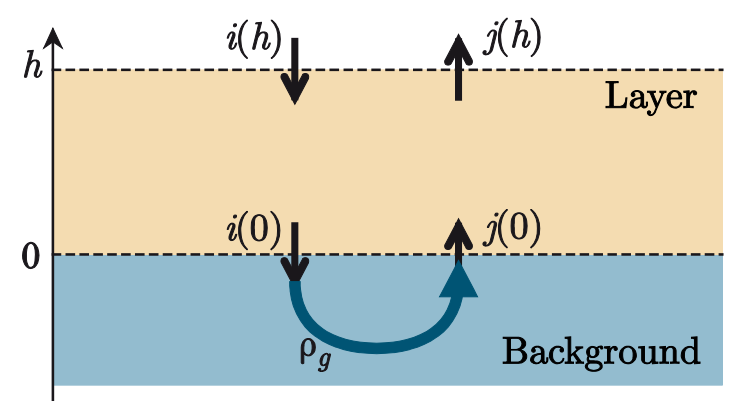

Figure 2: Upwards and downwards fluxes in a layer in optical contact with a background.

The boundary condition at $z=0$ can be written

$$
j(0)=\rho_{g} i(0)
$$

Substituting equation (24) in equation (22) yields:

$$
\rho=\frac{j(h)}{i(h)}=\frac{\left(1-a \rho_{g}\right) \sinh (b S h)+b \rho_{g} \cosh (b S h)}{\left(a-\rho_{g}\right) \sinh (b S h)+b \cosh (b S h)}
$$

In the case where $b S h \neq 0$, equation (25) can be written:

$$
\rho=\frac{1-\rho_{g}[a-b \operatorname{coth}(b S h)]}{a-\rho_{g}+b \operatorname{coth}(b S h)}
$$

Equation (26) is the hyperbolic solution of the Kubelka-Munk equations. In absence of scattering ( $S=0$ ), equation (23) yields: 


$$
\rho=\rho_{g} e^{-2 K h}
$$

Two special cases of interest are the reflectances $\rho_{0}$ and $\rho_{1}$ of a layer in optical contact with an ideal black background $\left(\rho_{g}=0\right)$, and with an ideal white background $\left(\rho_{g}=1\right)$ respectively. In the case of the black background, equation (26) becomes:

$$
\rho_{0}=\frac{\sinh (b S h)}{a \sinh (b S h)+b \cosh (b S h)}=\frac{1}{a+b \cdot \operatorname{coth}(b S h)}
$$

and in the case of the white background, it becomes:

$$
\rho_{1}=\frac{(1-a) \sinh (b S h)+b \cosh (b S h)}{(a-1) \sinh (b S h)+b \cosh (b S h)}=\frac{1-a+b \cdot \operatorname{coth}(b S h)}{a-1+b \cdot \operatorname{coth}(b S h)}
$$

Note that equation (28) can also be written

$$
b \cdot \operatorname{coth}(b S h)=\frac{1}{\rho_{0}}-a .
$$

and equation (29) can thus be written

$$
\rho_{1}=\frac{1-2 a+\left(1 / \rho_{0}\right)}{\left(1 / \rho_{0}\right)-1}
$$

or, using the defining expression (19) for $a$,

$$
a=\frac{K+S}{S}=\frac{1}{2}\left(1+\rho_{1}+\frac{1-\rho_{1}}{\rho_{0}}\right)
$$

This equation (32) which by definition relates $K$ and $S$ to $\rho_{1}$ and $\rho_{0}$ is an important result of the Kubelka-Munk theory.

Another important result is obtained by substituting relations (30) and (32) in equation (26):

$$
\rho=\frac{1-\rho_{g}\left[1+\rho_{1}\left(1-\frac{1}{\rho_{0}}\right)\right]}{\frac{1}{\rho_{0}}-\rho_{g}}=\frac{\rho_{0}\left(1-\rho_{g}\right)+\rho_{g} \rho_{1}\left(1-\rho_{0}\right)}{1-\rho_{g} \rho_{0}}
$$

This equation (33) allows for predicting the reflectance $\rho$ of the layer on a substrate of reflectance $\rho_{g}$, knowing $\rho_{0}$ and $\rho_{1}$.

In the case of paints, $\rho_{g}, \rho_{0}$ and $\rho$ are easily determined by experimental means. The paint is applied to a contrast card as shown in Figure 3, made of a light area of reflectance $\rho_{g}$ and a dark area that behaves like an ideal black background. The dark area covered by paint directly provides the reflectance $\rho_{0}$. The light area covered by paint has a reflectance $\rho$. Once $\rho_{0}, \rho_{g}$ 
and $\rho$ have been measured, we can deduce the reflectance $\rho_{1}$ of the paint applied on an ideal white background using the following formula, which comes from equation (33):

$$
\rho_{1}=\frac{\rho\left(1-\rho_{0} \rho_{g}\right)-\rho_{0}\left(1-\rho_{g}\right)}{\rho_{g}\left(1-\rho_{0}\right)}
$$

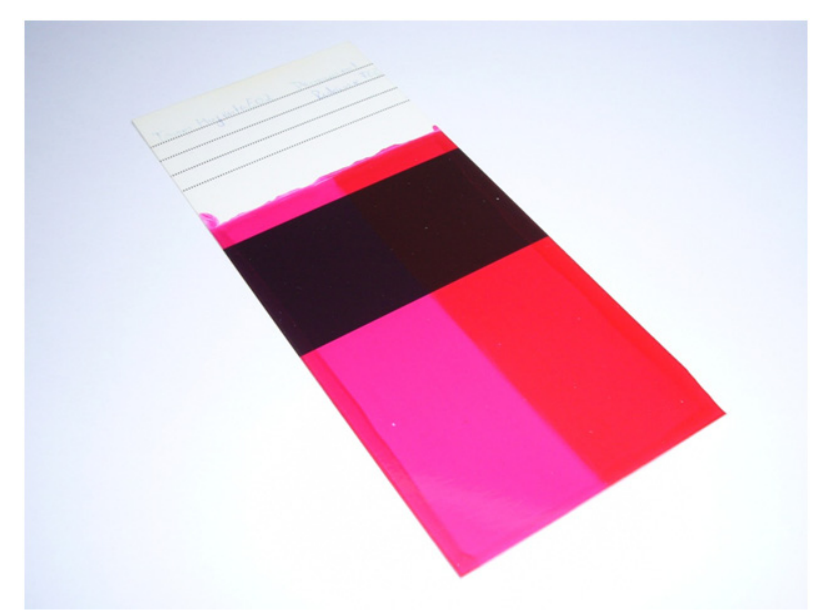

Figure 3: Contrast card with white and black areas used to determine $\rho_{1}$ and $\rho_{0}$.

Note that the reflectances $\rho, \rho_{0}, \rho_{1}$, and $\rho_{g}$ are all functions of wavelength.

\subsection{Intrinsic transmittance of a layer with defined thickness}

The intrinsic transmittance of the layer of thickness $h$, defined by equation (16), can be expanded as follows, according to equation (22):

$$
\tau_{i}=\left(\frac{i(0)}{i(h)}\right)_{j(0)=0}=\frac{2 b}{(a+b) e^{b S h}-(a-b) e^{-b S h}}=\frac{b}{a \sinh (b S h)+b \cosh (b S h)}
$$

\subsection{Infinitely thick layer}

A layer of thickness $h$ can be considered as "infinitely thick" when adding another layer of the same medium with finite thickness $X$ does not modify its body reflectance, denoted as $\rho_{\infty}$. One can thus write: 


$$
\begin{aligned}
\left(\begin{array}{c}
j(X+h) \\
i(X+h)
\end{array}\right) & =\mathbf{V} \cdot\left(\begin{array}{cc}
e^{v_{1}(X+h)} & 0 \\
0 & e^{v_{2}(X+h)}
\end{array}\right) \cdot \mathbf{V}^{-1} \cdot\left(\begin{array}{c}
j(0) \\
i(0)
\end{array}\right) \\
& =\mathbf{V} \cdot\left(\begin{array}{cc}
e^{v_{1} X} & 0 \\
0 & e^{v_{2} X}
\end{array}\right) \cdot\left(\begin{array}{cc}
e^{v_{1} h} & 0 \\
0 & e^{v_{2} h}
\end{array}\right) \cdot \mathbf{V}^{-1} \cdot\left(\begin{array}{c}
j(0) \\
i(0)
\end{array}\right) \\
& =\mathbf{V} \cdot\left(\begin{array}{cc}
e^{v_{1} X} & 0 \\
0 & e^{v_{2} X}
\end{array}\right) \cdot \mathbf{V}^{-1} \cdot\left(\begin{array}{c}
j(h) \\
i(h)
\end{array}\right) \\
& =\mathbf{V} \cdot\left(\begin{array}{cc}
e^{v_{1} X} & 0 \\
0 & e^{v_{2} X}
\end{array}\right) \cdot \mathbf{V}^{-1} \cdot\left(\begin{array}{c}
\rho_{\infty} i(h) \\
i(h)
\end{array}\right) \\
& =\mathbf{M}_{K, S, X} \cdot i(h)\left(\begin{array}{c}
\rho_{\infty} \\
1
\end{array}\right)
\end{aligned}
$$

Furthermore, one has:

$$
\left(\begin{array}{c}
j(X+h) \\
i(X+h)
\end{array}\right)=\left(\begin{array}{c}
\rho_{\infty} \cdot i(X+h) \\
i(X+h)
\end{array}\right)=i(X+h)\left(\begin{array}{c}
\rho_{\infty} \\
1
\end{array}\right)
$$

which therefore means, by identification of equations (36) and (37)

$$
\mathbf{M}_{K, S, X} \cdot i(h)\left(\begin{array}{c}
\rho_{\infty} \\
1
\end{array}\right)=i(X+h)\left(\begin{array}{c}
\rho_{\infty} \\
1
\end{array}\right)
$$

In linear algebra, equation (38) means that the column vector $\left(\rho_{\infty}, 1\right)$ is an eigenvector of $\mathbf{M}_{K, S, X}$ attached to the positive eigenvalue $\lambda_{2}=\sqrt{K^{2}+2 K S}$. Another eigenvector attached to this eigenvalue is $\left(S,(K+S)+\sqrt{K^{2}+2 K S}\right)$. The two vectors being collinear, one thus has

$$
\operatorname{det}\left(\begin{array}{cc}
\rho_{\infty} & S \\
1 & (K+S)+\sqrt{K^{2}+2 K S}
\end{array}\right)=0
$$

which yields the following expression for $\rho_{\infty}$ as a function of $K$ and $S$, well-known in the literature [9],

$$
\rho_{\infty}=1+\frac{K}{S}-\sqrt{\left(\frac{K}{S}\right)^{2}+2 \frac{K}{S}}
$$

Following equation (39), the ratio of $K$ to $S$ can be expressed as a function of $\rho_{\infty}$ :

$$
\frac{K}{S}=\frac{\left(1-\rho_{\infty}\right)^{2}}{2 \rho_{\infty}}
$$


Equation (40) is the most famous result from the Kubelka-Munk theory, widely used in the paper and textile industry. Other important results introduced above are listed in the next section.

\subsection{Most important formulas}

The Table below summarizes the most important formulas issued form the Kubelka-Munk theory. 
Table 1: Remarkable results from Kubelka-Munk theory

\begin{tabular}{|c|c|}
\hline $\begin{array}{l}\text { Parameter } a \text { as a function of the absorption coefficient } K \text { and } \\
\text { the scattering coefficient } S\end{array}$ & $a=\frac{K+S}{S}$ \\
\hline Parameter $b$ & $b=\sqrt{a^{2}-1}=\frac{1}{S} \sqrt{K^{2}+2 K S}$ \\
\hline $\begin{array}{l}\text { Hyperbolic solution of the Kubelka-Munk equations, for a } \\
\text { layer on a background with reflectance } \rho_{g} \text { [equation (26)] }\end{array}$ & $\rho=\frac{1-\rho_{g}(a-b \operatorname{coth}(b S h))}{a-\rho_{g}+b \operatorname{coth}(b S h)}$ \\
\hline $\begin{array}{l}\text { Reflectance of a layer on an ideal black background } \\
\left(\rho_{g}=0\right) \text { [equation }(28) \text { ] }\end{array}$ & $\rho_{0}=\frac{1}{a+b \cdot \operatorname{coth}(b S h)}$ \\
\hline $\begin{array}{l}\text { Reflectance of a layer on an ideal white background } \\
\left(\rho_{g}=1\right) \text { [equation (29)] }\end{array}$ & $\rho_{1}=\frac{1-a+b \cdot \operatorname{coth}(b S h)}{a-1+b \cdot \operatorname{coth}(b S h)}$ \\
\hline Parameter $a$ as a function of $\rho_{0}$ and $\rho_{1}$ [equation (32)] & $a=\frac{K+S}{S}=\frac{1}{2}\left(1-\frac{\rho_{1}-1}{\rho_{0}}\right)$ \\
\hline $\begin{array}{l}\text { Reflectance of a layer on a background with reflectance } \rho_{g} \\
\text { as a function of } \rho_{0} \text { and } \rho_{1} \text { [equation (33)] }\end{array}$ & $\rho=\frac{\rho_{0}\left(1-\rho_{g}\right)+\rho_{1} \rho_{g}\left(1-\rho_{0}\right)}{1-\rho_{0} \rho_{g}}$ \\
\hline $\begin{array}{l}\text { Reflectance of a layer on an ideal white background as a } \\
\text { function of the reflectance } \rho \text { of this layer on some } \\
\text { background with reflectance } \rho_{g} \text { and of } \rho_{0} \text { [equation (34)] }\end{array}$ & $\rho_{1}=\frac{\rho\left(1-\rho_{0} \rho_{g}\right)-\rho_{0}\left(1-\rho_{g}\right)}{\rho_{g}\left(1-\rho_{0}\right)}$ \\
\hline Intrinsic transmittance $\tau_{i}$ of a layer [equation (35)] & $\tau_{i}=\frac{b}{a \sinh (b S h)+b \cosh (b S h)}$ \\
\hline $\begin{array}{l}\text { Reflectance } \rho_{\infty} \text { of an infinitely thick layer as a function of } \\
\text { the } K / S \text { ratio [equation (39)] }\end{array}$ & $\rho_{\infty}=a-b=1+\frac{K}{S}-\sqrt{\left(\frac{K}{S}\right)^{2}+2 \frac{K}{S}}$ \\
\hline$K / S$ ratio as a function of $\rho_{\infty}$ [equation (40)] & $\frac{K}{S}=\frac{\left(1-\rho_{\infty}\right)^{2}}{2 \rho_{\infty}}$ \\
\hline
\end{tabular}

\subsection{Matrix formulation of the Saunderson correction}

Section 7.5 in [HDI062] introduced the Saunderson correction that accounts for the multiple internal reflections that occur at the interface located at the altitude $h$ between air (medium 0 ) and the layer (medium 1), assumed to be flat. The equations (120) in [HDI062] written at altitude $h$ can also be turned into a matrix form, by recalling that the reflectance for diffuse 
light of the interface at the air side is $r_{01}$ defined by equation (37) in [HDI062] as a function of the index of the medium; the transmittance from medium 0 to medium 1 is $t_{01}=1-r_{01}$, the transmittance from the medium 1 to the medium 0 is

$$
t_{10}=\frac{1}{n^{2}} t_{01}
$$

where $n$ is the relative refractive index of the media, and the reflectance at the side of medium 1 is $r_{10}=1-t_{10}$.

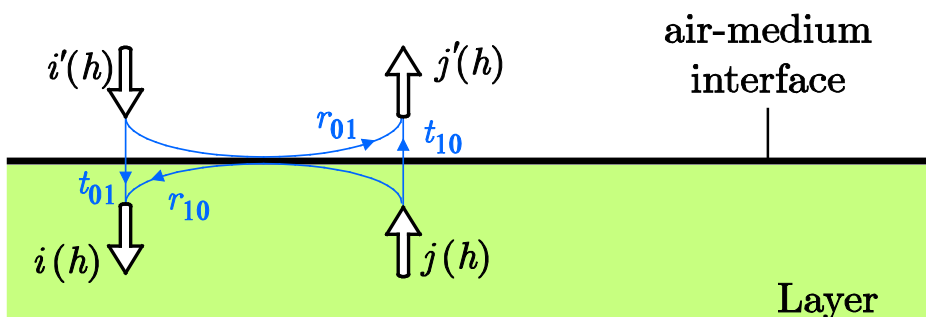

Figure 4: Reflection and transmission of diffuse fluxes at the air-layer interface.

Let us denote as $i^{\prime}(h)$ and $j^{\prime}(h)$ the respectively downward, and upward fluxes in air (Figure 4). The downward and upward fluxes in the layer are $i(h)$ and $j(h)$. One can write the two following equations

$$
\left\{\begin{array}{l}
i(h)=t_{01} i^{\prime}(h)+r_{10} j(h) \\
j^{\prime}(h)=r_{01} i^{\prime}(h)+t_{10} j(h)
\end{array}\right.
$$

which may written in a matrix form:

$$
\left(\begin{array}{cc}
0 & t_{01} \\
1 & -r_{01}
\end{array}\right)\left(\begin{array}{l}
j^{\prime}(h) \\
i^{\prime}(h)
\end{array}\right)=\left(\begin{array}{cc}
-r_{10} & 1 \\
t_{10} & 0
\end{array}\right)\left(\begin{array}{l}
j(h) \\
i(h)
\end{array}\right)
$$

This vector equation yields:

$$
\left(\begin{array}{l}
j^{\prime}(h) \\
i^{\prime}(h)
\end{array}\right)=\mathbf{f}_{01}\left(\begin{array}{l}
j(h) \\
i(h)
\end{array}\right)
$$

with

$$
\mathbf{f}_{01}=\frac{1}{t_{01}}\left(\begin{array}{cc}
t_{01} t_{10}-r_{01} r_{10} & r_{01} \\
-r_{10} & 1
\end{array}\right)
$$


or equivalently, according to the relations between reflectances and transmittances of the interface:

$$
\mathbf{f}_{01}=\frac{1}{1-r_{01}}\left(\begin{array}{cc}
\frac{1-r_{01}\left(1+n^{2}\right)}{n^{2}} & r_{01} \\
\frac{\left(1-r_{01}\right)}{n^{2}}-1 & 1
\end{array}\right)
$$

When considering the layer and its interface with air, the combination of the equations (44) and (8) yields:

$$
\left(\begin{array}{l}
j^{\prime}(h) \\
i^{\prime}(h)
\end{array}\right)=\mathbf{f}_{01} \cdot \mathbf{M}_{K, S, h} \cdot\left(\begin{array}{c}
j(0) \\
i(0)
\end{array}\right)
$$

We see through this equation (47) that the matrix model enables expressing the superposition of two reflecting-transmitting components (here a flat interface and a diffusing layer) by the product of matrices attached to each of them. This interesting property will be developed and generalized in Section 3, where the matrices are given the name of "transfer matrices".

Note that the reflectances and transmittances of a rough interface are numerically very close to those of a flat interface, tabulated in Appendix B of [HDI062] for various refractive indices. Note also that equation (47) applies to diffuse fluxes only. Most measuring devices use at least one collimated beam of light. This needs to be accounted for when considering a particular measuring geometry.

\subsection{Limitations of the Kubelka-Munk model}

In order to illustrate the limits imposed by the assumptions made in the Kubelka-Munk framework, let us consider two extreme cases where reflectance $\rho \approx 0$ and transmittance $t \approx 0$.

A dark piece of film has a high absorption $(K \gg 0)$ and no scattering $S=0$. Its high absorption makes it almost opaque. However, it can be used to observe very bright objects, as for instance, the filament of a light bulb. The absence of scattering yields a sharp picture. A piece of black cardboard has a high absorption $K \gg 0$ and a high scattering $S \gg 0$. An observer will not be able to distinguish a sharp picture while observing a bright object behind the piece of cardboard.

The Kubelka-Munk theory successfully predicts the reflectance and transmittance of a material assuming the light fluxes remain Lambertian through the medium. If this assumption 
does not hold, the absorption and scattering coefficients computed from the reflectance and the transmittance does not correspond to the properties of the medium [10]. The KubelkaMunk equations predict large absorption and scattering coefficients in both cases described above.

This limitation makes the Kubelka-Munk theory inapplicable to media where light fluxes are not always lambertian. The theory can be applied to paint where scattering is strong and guarantees the lambertian distribution, but not to inks where transparency favors collimated light fluxes.

Let us consider another special case where non-absorbing transparent film $(K=0)$ contains small scattering particles that induce forward scattering. Such a film looks blurry. An observer would not be able to distinguish a sharp picture when putting this film in front of him. Surprisingly, the scattering coefficient $S$ would be zero since it represents backscattering only. Actually, this scattering coefficient is not exactly zero but very small. As the thickness of the layer tends to infinity, backscattered flux produced by multiple scattering may become well perceptible.

\section{MATRIX MODEL FOR STACKS OF DIFFUSING LAYERS}

In 1954, Kubelka published an extension of the Kubelka-Munk model in case of nonhomogeneous layers [6]. When several layers are stacked together, Kubelka derived expressions for the reflectance and transmittance of the stack as functions of the individual reflectances and transmittances of the layers. These expressions were first derived for two layers according to a simple method based on geometrical series. However, this method becomes rapidly tedious when the number of layer increases. Pursuing the matrix formulation introduced in Section 2 in the context of the Kubelka-Munk model, we propose here to generalize the concept of transfer matrices relating the upward and downward fluxes entering and exiting the layers, which appeared through the definition of matrix $\mathbf{M}_{K, S, h}$ in equation (8). In this section, we also consider, like Kubelka in his paper, non-homogenous layers, i.e. layers having different reflectance at their front and back sides, which is not allowed in the original Kubelka-Munk model.

Throughout this section, the following notations are used: the layers are labeled with a number $k=1,2, \ldots$ incremented in the order of superposition. The reflectances of their front face ("front reflectance") and back face ("back reflectance") are denoted respectively as $\rho_{k}$ 
and $\rho_{k}^{\prime}$. These two reflectances are a priori different due for example to a gradient of absorption coefficient across the thickness of the layer. Their transmittances are denoted as $\tau_{k}$ and $\tau_{k}^{\prime}$ when light transits from front to back ("forward transmittance"), and respectively from back to front ("backward transmittance"). In practice, the transmittances $\tau_{k}$ and $\tau_{k}^{\prime}$ are identical according to Kubelka's nonpolarity principle for transmittance. However, we propose to maintain a distinction between the two notations. Downward and upward fluxes are respectively denoted using letters $i$ and $j$. Subscripts $k=0,1, \ldots$ correspond to their position in the stack: 0 when the flux is in the surrounding medium at the front side, 1 when it is between layers 1 and 2, and so on. Figure 5 shows an example for two layers.

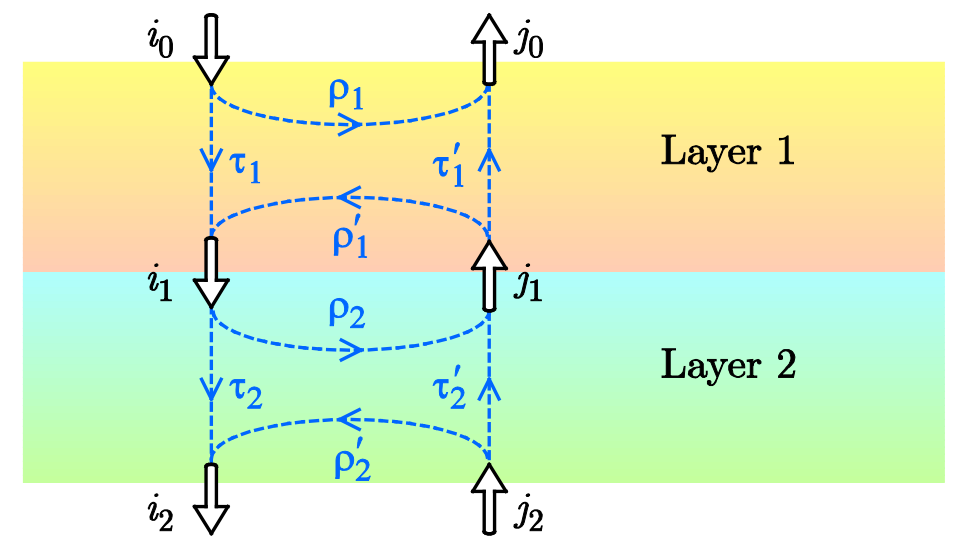

Figure 5: Kubelka's model described the reflections and transmissions (dashed arrows) of Lambertian fluxes (white arrows) between nonsymmetric diffusing layers.

\subsection{Transfer matrices}

Let us consider one layer, for example Layer 1 in Figure 5. By describing how each flux contributes to each other flux, we obtain the following relations:

$$
\begin{aligned}
& j_{0}=\rho_{1} i_{0}+\tau_{1}^{\prime} j_{1} \\
& i_{1}=\tau_{1} i_{0}+\rho_{1}^{\prime} j_{1}
\end{aligned}
$$

which may also be written

$$
\left(\begin{array}{cc}
0 & \tau_{1} \\
1 & -\rho_{1}
\end{array}\right)\left(\begin{array}{l}
j_{0} \\
i_{0}
\end{array}\right)=\left(\begin{array}{cc}
-\rho_{1}^{\prime} & 1 \\
\tau_{1}^{\prime} & 0
\end{array}\right)\left(\begin{array}{l}
j_{1} \\
i_{1}
\end{array}\right)
$$

or

$$
\left(\begin{array}{c}
j_{0} \\
i_{0}
\end{array}\right)=\frac{1}{\tau_{1}}\left(\begin{array}{cc}
\tau_{1} \tau_{1}^{\prime}-\rho_{1} \rho_{1}^{\prime} & \rho_{1} \\
-\rho_{1}^{\prime} & 1
\end{array}\right)\left(\begin{array}{l}
j_{1} \\
i_{1}
\end{array}\right)
$$


The matrix in equation (51), denoted as $\mathbf{M}_{1}$, is the transfer matrix attached to layer 1

$$
\mathbf{M}_{1}=\frac{1}{\tau_{1}}\left(\begin{array}{cc}
\tau_{1} \tau_{1}^{\prime}-\rho_{1} \rho_{1}^{\prime} & \rho_{1} \\
-\rho_{1}^{\prime} & 1
\end{array}\right)
$$

This line of reasoning stands for any layer (or interface, see Section 2.7). It also stands for a group of layers (or of layers and interfaces), for example for the two layers described in Figure 5. Let us denote as $\rho_{12}$ their global front reflectance, $\tau_{12}$ their forward transmittance, $\rho_{12}^{\prime}$ their back transmittance and $\tau_{12}^{\prime}$ their upward transmittance. We have:

$$
\left(\begin{array}{l}
j_{0} \\
i_{0}
\end{array}\right)=\frac{1}{\tau_{12}}\left(\begin{array}{cc}
\tau_{12} \tau_{12}^{\prime}-\rho_{12} \rho_{12}^{\prime} & \rho_{12} \\
-\rho_{12}^{\prime} & 1
\end{array}\right)\left(\begin{array}{l}
j_{2} \\
i_{2}
\end{array}\right)
$$

The matrix in equation (53), denoted $\mathbf{M}_{12}$, is the transfer matrix attached to the two layers. We can also write the equalities:

$$
\left(\begin{array}{c}
j_{0} \\
i_{0}
\end{array}\right)=\mathbf{M}_{1}\left(\begin{array}{l}
j_{1} \\
i_{1}
\end{array}\right)=\mathbf{M}_{1} \cdot \mathbf{M}_{2}\left(\begin{array}{l}
j_{2} \\
i_{2}
\end{array}\right)
$$

which show that the transfer matrix of two layers is the product of the individual transfer matrices of the layers. This was already noticed in Section 2.7, equation (47). Note that the left-to-right position of the matrices in the product reproduces the forward position of the corresponding layers. This also holds for many layers, i.e. for any integer $k$

$$
\mathbf{M}_{123 \ldots k}=\mathbf{M}_{1} \mathbf{M}_{2} \mathbf{M}_{3} \ldots \mathbf{M}_{k}
$$

This matrix product for successive layers extends the product of the transmittances of successive nonscattering layers according to Beer's law.

Every transfer matrix, even when obtained by a product of transfer matrices, has the same structure of the form:

$$
\mathbf{M}=\frac{1}{\tau}\left(\begin{array}{cc}
\tau \tau^{\prime}-\rho \rho^{\prime} & \rho \\
-\rho^{\prime} & 1
\end{array}\right)
$$

From a given transfer matrix $\mathbf{M}=\left\{m_{p q}\right\}$, we retrieve the front reflectance $\rho$, back reflectance $\rho^{\prime}$, downward transmittance $\tau$ and upward transmittance $\tau^{\prime}$ in the following way:

$$
\begin{aligned}
& \rho=m_{12} / m_{22} \\
& \tau=1 / m_{22} \\
& \rho^{\prime}=-m_{21} / m_{22} \\
& \tau^{\prime}=\operatorname{det}(\mathbf{M}) / m_{22}
\end{aligned}
$$

where the symbol 'det' denotes the determinant of the matrix. 
Note that similar transfer matrices are used in wave optics to model the propagation of electric fields [12] or incoherent light beams [13, 14] in thin multilayers. Compared to the geometrical series, the matrix method becomes very advantageous as soon as more than two layers are superposed.

\subsection{Examples}

Let us come back to the example of two layers and expand the transfer matrix $\mathbf{M}_{12}$

$$
\begin{aligned}
\mathbf{M}_{12} & =\mathbf{M}_{1} \cdot \mathbf{M}_{2} \\
& =\frac{1}{\tau_{1} \tau_{2}}\left(\begin{array}{cc}
\tau_{1} \tau_{1}^{\prime}-\rho_{1} \rho_{1}^{\prime} & \rho_{1} \\
-\rho_{1}^{\prime} & 1
\end{array}\right) \cdot\left(\begin{array}{cc}
\tau_{2} \tau_{2}^{\prime}-\rho_{2} \rho_{2}^{\prime} & \rho_{2} \\
-\rho_{2}^{\prime} & 1
\end{array}\right) \\
& =\frac{1}{\tau_{1} \tau_{2}}\left(\begin{array}{cc}
-\rho_{1} \rho_{2}^{\prime}+\left(\tau_{1} \tau_{1}^{\prime}-\rho_{1} \rho_{1}^{\prime}\right)\left(\tau_{2} \tau_{2}^{\prime}-\rho_{2} \rho_{2}^{\prime}\right) & \rho_{1}+\rho_{2}\left(\tau_{1} \tau_{1}^{\prime}-\rho_{1} \rho_{1}^{\prime}\right) \\
-\rho_{2}^{\prime}-\rho_{1}^{\prime}\left(\tau_{2} \tau_{2}^{\prime}-\rho_{2} \rho_{2}^{\prime}\right) & 1-\rho_{1}^{\prime} \rho_{2}
\end{array}\right)
\end{aligned}
$$

By applying formulas (57) on the matrix $\mathbf{M}_{12}$ expanded in equation (58), we retrieve the Kubelka's formulas expressing the reflectances and transmittances of two layers as a function of the individual reflectances and transmittances of the layers.

$$
\begin{gathered}
\rho_{12}=\frac{m_{12}}{m_{22}}=\rho_{1}+\frac{\tau_{1} \tau_{1}^{\prime} \rho_{2}}{1-\rho_{1}^{\prime} \rho_{2}}, \\
\tau_{12}=\frac{1}{m_{22}}=\frac{\tau_{1} \tau_{2}}{1-\rho_{1}^{\prime} \rho_{2}}, \\
\rho_{12}^{\prime}=\frac{-m_{2}}{m_{22}}=\rho_{2}^{\prime}+\frac{\tau_{2} \tau_{2}^{\prime} \rho_{1}^{\prime}}{1-\rho_{1}^{\prime} \rho_{2}},
\end{gathered}
$$

and

$$
\tau_{12}^{\prime}=\frac{\operatorname{det}\left(\mathbf{M}_{12}\right)}{m_{22}}=\frac{\tau_{1}^{\prime} \tau_{2}^{\prime}}{1-\rho_{1}^{\prime} \rho_{2}}
$$

These formulas are generally derived by reducing the geometrical series [6]. The individual reflectances and transmittances of layers, if they are homogenous, may be computed using the Kubelka-Munk mode and in any case, they can also be measured. Most of the time, they depend on wavelength. In this case, the matrix model, as well as Kubelka's formulas, applies for each waveband.

In a diffusing multilayer, the interfaces between layers have no optical effect if the layers have the same refractive index. Otherwise, light reflections and refractions occur at the interfaces and modify the flux transfers between the layers. We thus proceed as in Section 2.7, 
by using the transfer matrices attached to the interfaces between media with refractive indices $n_{k}$ (on the front face) and $n_{l}$ (on the back face), similarly defined as in equation (45):

$$
\mathbf{f}_{k l}=\frac{1}{t_{k l}}\left(\begin{array}{cc}
t_{k l} t_{l k}-r_{k l} r_{l k} & r_{k l} \\
-r_{l k} & 1
\end{array}\right)
$$

Let us consider for example a symmetric layer (medium 1 with reflectance $\rho$ and transmittance $\tau$ ) bordered on its two faces by air (medium 0 ). The incoming lights at front and back sides are Lambertian. The transfer matrix attached to the layer with interfaces is

$$
\mathbf{M}=\mathbf{f}_{01} \cdot \mathbf{M}_{1} \cdot \mathbf{f}_{10}=\left(\begin{array}{ll}
m_{11} & m_{12} \\
m_{21} & m_{22}
\end{array}\right)
$$

where $\mathbf{M}_{1}$ denotes the matrix attached to the layer defined by equation (56), and $\mathbf{f}_{01}$ and $\mathbf{f}_{10}$ denote the matrices attached to the front, respectively back interfaces, defined by equation (63). After computation, we obtain:

$$
m_{22}=\frac{1}{t_{01} \tau t_{10}}\left[1-r_{10}\left(\rho+\rho^{\prime}\right)-r_{10}^{2}\left(\tau \tau^{\prime}-\rho \rho^{\prime}\right)\right]
$$

and

$$
m_{12}=r_{01}+\frac{1}{\tau}\left[\rho+r_{10}\left(\tau \tau^{\prime}-\rho \rho^{\prime}\right)\right]
$$

According to equations (57), we retrieve the well known formulas for the reflectance $R$ and the transmittance $T$ of the layer with its interfaces with air [15]

$$
\begin{gathered}
T=\frac{1}{m_{22}}=\frac{t_{01} \tau t_{10}}{1-r_{10}\left(\rho+\rho^{\prime}\right)-r_{10}^{2}\left(\tau \tau^{\prime}-\rho \rho^{\prime}\right)} \\
R=\frac{m_{12}}{m_{22}}=r_{01}+t_{01} t_{10} \frac{\rho+r_{10}\left(\tau \tau^{\prime}-\rho \rho^{\prime}\right)}{1-r_{10}\left(\rho+\rho^{\prime}\right)-r_{10}^{2}\left(\tau \tau^{\prime}-\rho \rho^{\prime}\right)}
\end{gathered}
$$

We may generalize the method for two layers with different refractive indices, as represented in Figure 6. Once again, light sources are Lambertian at both sides. Transfer matrices $\mathbf{M}_{1}$ and $\mathbf{M}_{2}$ defined in equation (52) are attached to the layers and transfer matrices $\mathbf{f}_{01}, \mathbf{f}_{12}$ and $\mathbf{f}_{23}$ defined in equation (63) are attached to the front, middle and back interfaces reflectively. The transfer matrix of the stack is thus given by:

$$
\mathbf{M}=\mathbf{f}_{01} \cdot \mathbf{M}_{1} \cdot \mathbf{f}_{12} \cdot \mathbf{M}_{2} \cdot \mathbf{f}_{23}
$$

The reflectances and transmittances of the multilayer are then deduced from the entries of $\mathbf{M}$ according to the formulas (57). 


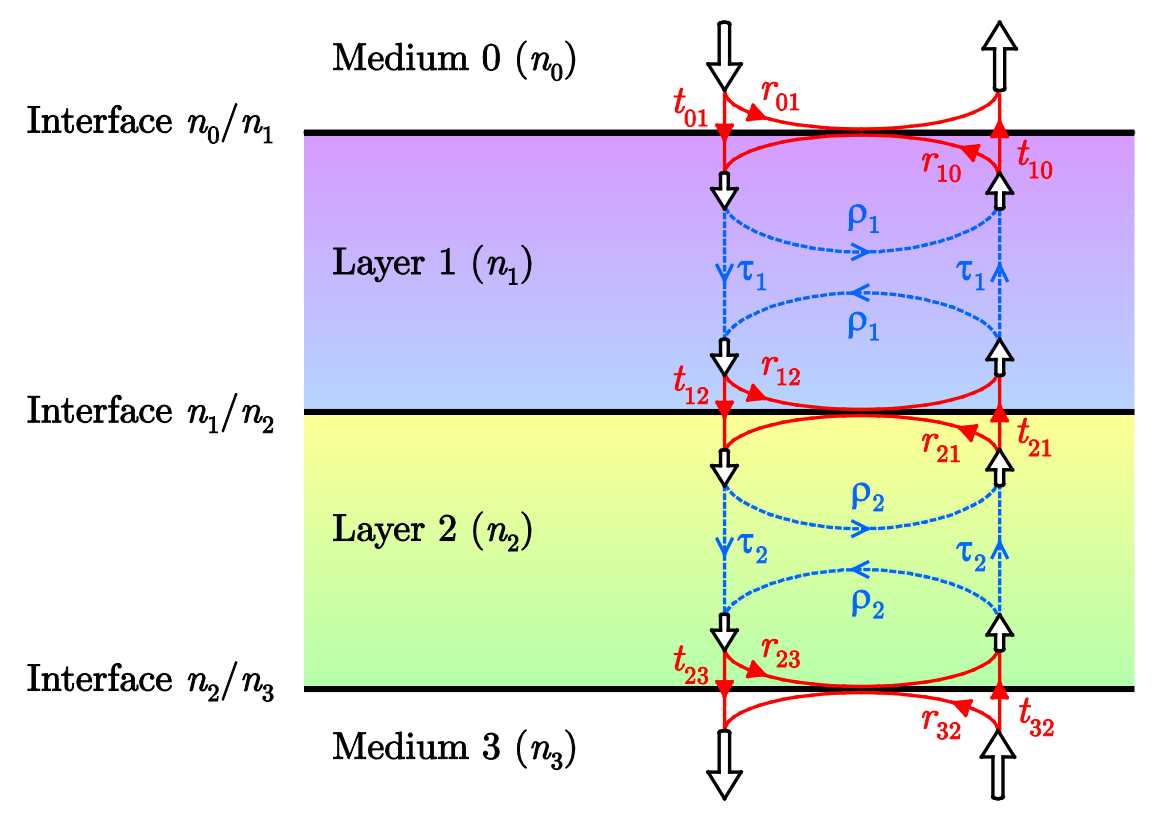

Figure 6: Flux transfers between diffusing layers (dashed blued arrows) and by interfaces (solid red arrows).

\section{MATRIX MODEL FOR STACKS OF FILMS}

Although nonscattering media are not reflective by themselves, their surface reflects light. When several layers with distinct indices are stacked together, light is thus reflected multiple times. We will see that transfer matrices can also be defined in this context. Layer thicknesses are assumed much thicker than the coherence length of light, which thus avoid interference effects and enable using laws of geometrical optics. The main difference between nonscattering and scattering media is the fact that light propagates along straight lines, or broken straight lines due to refraction at the interfaces, with orientations well determined according to Snell's law (Figure 7). These orientations determine the reflectance and transmittance at the interfaces, as well as their attenuation while crossing the layers. This is the reason why nonscattering layers, multilayers and flat interfaces will be first characterized by an angular transfer matrix, based on angular reflectance and transmittance functions. When the incident light is diffuse, we can integrate these functions over the set of incident angles. 


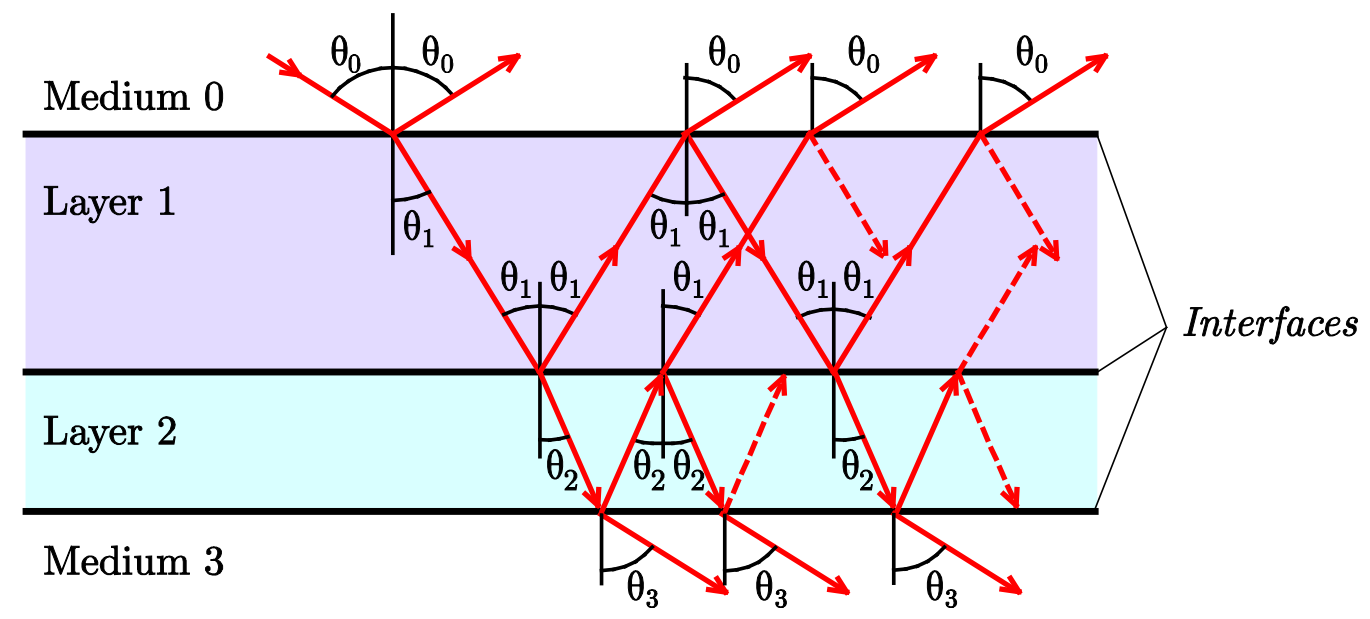

Figure 7. In a nonscattering multilayer, the direction of the light rays in each layer is fixed. It depends only on the direction of the incident light, $\theta_{0}$, and on the refractive index of the layer.

The reflectance of nonscattering multilayers also depends on polarization of light due to the polarization-dependant Fresnel formulae. If the incident light is linearly polarized in the incidence plane or perpendicularly to it, this polarization is maintained in the whole multiple reflection process and determines which Fresnel formula must be used for the reflectances contained within the reflectance and transmittance expressions. In the case of natural light, which is the type of light emitted by most light sources, we assume that the two linearly polarized components flow in parallel, independently of each other and with equal initial power (see [HDI062], Section 2.2). The multilayer's reflectance and transmittance is the average of the reflectances and transmittances attached to the parallel $(p)$ and perpendicular $(s)$ polarizations. This may be written by the following equation

$$
X=\frac{f\left(R_{p}\right)+f\left(R_{s}\right)}{2}
$$

where $X$ denotes a multilayer reflectance (or transmittance) for natural light, $f$ the analytical reflectance (or transmittance) expression being a function of Fresnel reflectance $R_{p}$ for the parallel polarization or $R_{S}$ for the perpendicular polarization.

Nevertheless, many nonscattering materials such as polymers have a strong optical anisotropy which decreases the degree of polarization of light. In practice, it is more exact to consider that light remains unpolarized along the whole multiple reflection process and to insert the Fresnel reflectance or transmittance for unpolarized light, the average of the reflectances or transmittances for $\mathrm{p}$ - and s-polarized lights, in the multilayers' reflectance and transmittance expressions which thus becomes 


$$
X=f\left(\frac{R_{p}+R_{s}}{2}\right)
$$

We will consider hereinafter this latter case. In addition to being simpler because it prevents having to expand expressions for each polarization and to average them, this choice is justified by the fact that polymers are used in our experimental applications. Nevertheless, it is not an issue to follow one approach or to the other: the model in itself provides only the $f$ function.

\subsection{Single film at normal incidence}

Let us first consider the simple case of a nonscattering film illuminated by collimated light at normal incidence. The film is made of nonscattering medium with index $n_{1}$. Its transmittance at normal incidence is denoted as $T_{1}$. It is bordered by a clear medium with index $n_{0}$ at front side, e.g. air, and by a possibly different medium with index $n_{2}$ at back side, e.g. water. Regarding the Fresnel reflectances of the interfaces, similar notations are used as in [HDI062], Section 3. Since light is normal to the interfaces, we have:

$$
R_{j k}(0)=R_{k j}(0)=\left(\frac{n_{k}-n_{j}}{n_{k}+n_{j}}\right)^{2}
$$

and

$$
T_{k j}(0)=T_{j k}(0)=1-R_{j k}(0) .
$$

In this section, the short notation $R_{j k}$ for $R_{j k}(0)$ is used.

Figure 8 shows the flux transfers between the different media. As for a diffusing layer [equations (48) to (51)], we may write the following matrix equation for an interface between media $j$ and $k=j+1$

$$
\left(\begin{array}{cc}
0 & T_{j k} \\
1 & -R_{j k}
\end{array}\right)\left(\begin{array}{c}
J_{j} \\
I_{j}^{\prime}
\end{array}\right)=\left(\begin{array}{cc}
1 & -R_{k j} \\
0 & T_{k j}
\end{array}\right)\left(\begin{array}{c}
J_{k}^{\prime} \\
I_{k}
\end{array}\right)
$$

or, since $T_{j k}$ cannot be zero at normal incidence,

$$
\left(\begin{array}{c}
J_{j} \\
I_{j}^{\prime}
\end{array}\right)=\frac{1}{T_{j k}}\left(\begin{array}{cc}
T_{j k} T_{k j}-R_{j k} R_{k j} & R_{j k} \\
-R_{k j} & 1
\end{array}\right)\left(\begin{array}{c}
J_{k}^{\prime} \\
I_{k}
\end{array}\right)
$$

The matrix in equation (70), denoted $\mathbf{F}_{j k}$, is the transfer matrix attached to the interface:

$$
\mathbf{F}_{j k}=\frac{1}{T_{j k}}\left(\begin{array}{cc}
T_{j k} T_{k j}-R_{j k} R_{k j} & R_{j k} \\
-R_{k j} & 1
\end{array}\right)
$$




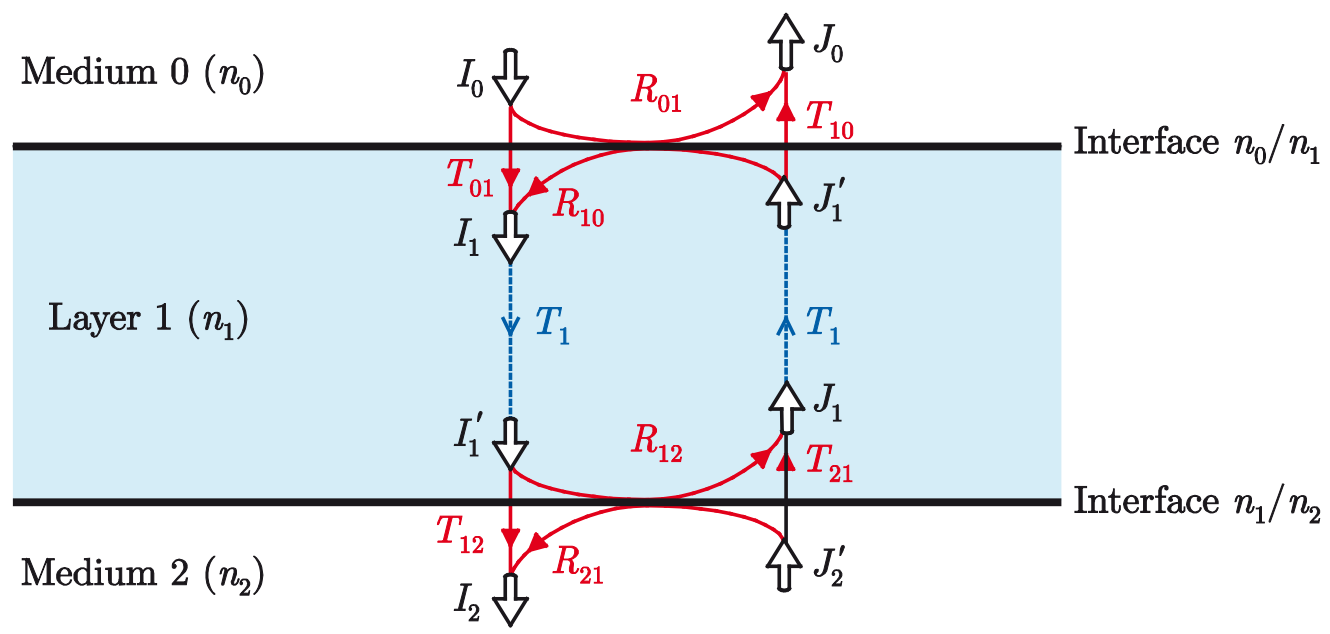

Figure 8. Flux transfers through the interfaces and nonscattering layer of a film of index $n_{1}$ bordered by media of index $n_{0}$ at the front side, and of index $n_{2}$ at the back side.

Note that using the relations (68) and (69), we can transform equation (71) into

$$
\mathbf{F}_{j k}=\frac{1}{1-R_{j k}}\left(\begin{array}{cc}
1-2 R_{j k} & R_{j k} \\
-R_{j k} & 1
\end{array}\right)
$$

Regarding the film bulk layer 2, denoting $I_{1}^{\prime}$ and $J_{1}^{\prime}$ the fluxes $I_{1}$ and $J_{1}$ after transmission across the layer, we have

$$
\left(\begin{array}{ll}
1 & 0 \\
0 & t
\end{array}\right)\left(\begin{array}{l}
J_{1}^{\prime} \\
I_{1}
\end{array}\right)=\left(\begin{array}{ll}
T_{1} & 0 \\
0 & 1
\end{array}\right)\left(\begin{array}{c}
J_{1} \\
I_{1}^{\prime}
\end{array}\right)
$$

i.e.,

$$
\left(\begin{array}{c}
J_{1}^{\prime} \\
I_{1}
\end{array}\right)=\mathbf{L}_{1}\left(\begin{array}{c}
J_{1} \\
I_{1}^{\prime}
\end{array}\right)
$$

where $\mathbf{L}_{1}$ is the transfer matrix attached to the layer, which depends upon orientation but not on polarization of light:

$$
\mathbf{L}_{t}=\frac{1}{T_{1}}\left(\begin{array}{rr}
T_{1}^{2} & 0 \\
0 & 1
\end{array}\right),
$$

Combining equations (70) and (73)

$$
\left(\begin{array}{c}
J_{0} \\
I_{0}
\end{array}\right)=\mathbf{F}_{01} \mathbf{L}_{1} \mathbf{F}_{12}\left(\begin{array}{c}
J_{2} \\
I_{2}
\end{array}\right)
$$

shows that the matrix product $\mathbf{F}_{01} \mathbf{L}_{1} \mathbf{F}_{12}$ is the transfer matrix of the film: 


$$
\mathbf{M}=\frac{1}{T_{01} T_{1} T_{12}}\left(\begin{array}{cc}
\left(T_{01}^{2}-R_{01}^{2}\right)\left(T_{12}^{2}-R_{12}^{2}\right) T_{1}^{2}-R_{01} R_{12} & R_{01}+R_{12}\left(T_{01}^{2}-R_{01}^{2}\right) T_{1}^{2} \\
-R_{23}-R_{01}\left(T_{12}^{2}-R_{12}^{2}\right) T_{1}^{2} & 1-R_{12} R_{01} T_{1}^{2}
\end{array}\right)
$$

We can retrieve the front and back reflectances and the forward and backward transmittances of the film using similar equations as (57). For example, the front reflectance of the film is

$$
R_{f}=\frac{m_{12}}{m_{22}}=R_{01}+\frac{T_{01}^{2} R_{12} T_{1}^{2}}{1-R_{12} R_{01} T_{1}^{2}}
$$

and its forward transmittance is

$$
T_{f}=\frac{1}{m_{22}}=\frac{T_{01} T_{1} T_{12}}{1-R_{12} R_{01} T_{1}^{2}}
$$

\subsection{Single film at oblique incidence}

At oblique incidence, the same line of reasoning applies, except that the orientation of light is different in the three media. However, in a given medium, all light components form the same angle with the normal, as shown in Figure 7. The orientation of fluxes $I_{0}$ and $J_{0}$ is $\theta_{0}$, the one of fluxes $I_{1}, I_{1}^{\prime}, J_{1}$ and $J_{1}^{\prime}$ is $\theta_{1}$ and the orientation of fluxes $I_{2}$ and $J_{2}$ is $\theta_{2}$. Writing Snell's sine law at the successive interfaces yields the following equality

$$
n_{0} \sin \theta_{0}=n_{1} \sin \theta_{1}=n_{2} \sin \theta_{2}
$$

from which we deduce that the angle $\theta_{k}$ in medium $k=1$ or 2 depends only on $\theta_{0}, n_{0}$ and $n_{k}$, not on the indices of the other medium:

$$
\theta_{k}=\arcsin \left(n_{0} \sin \theta_{0} / n_{k}\right)
$$

Equation (70) remains valid but with different definition for the transfer matrix of the interface, $\mathbf{F}_{j k}$, whose entries are now function of the angles $\theta_{j}$ (front reflectance, forward transmittance) and $\theta_{k}$ (back reflectance and backward transmittance):

$$
\mathbf{F}_{j k}\left(\theta_{j}\right)=\frac{1}{T_{j k}\left(\theta_{j}\right)}\left(\begin{array}{cc}
T_{j k}\left(\theta_{j}\right) T_{k j}\left(\theta_{k}\right)-R_{j k}\left(\theta_{j}\right) R_{k j}\left(\theta_{k}\right) & R_{j k}\left(\theta_{j}\right) \\
-R_{k j}\left(\theta_{k}\right) & 1
\end{array}\right)
$$

The properties of the Fresnel reflectance and transmittance formulas enable transforming the above equation into:

$$
\mathbf{F}_{j k}\left(\theta_{j}\right)=\frac{1}{1-R_{j k}\left(\theta_{j}\right)}\left(\begin{array}{cc}
1-2 R_{j k}\left(\theta_{j}\right) & R_{j k}\left(\theta_{j}\right) \\
-R_{j k}\left(\theta_{j}\right) & 1
\end{array}\right)
$$


Note that there is a computational problem in case of total reflection, i.e. when $R_{j k}\left(\theta_{j}\right)=1$. The way to work around this problem is to use an alternative definition of transfer matrices, presented in appendix, which avoids the division by the term $1-R_{j k}\left(\theta_{j}\right)$.

Regarding the layer, equation (73) also remains valid but, once again, the transfer matrix attached to the layer is modified in order to account for the orientation of light in the layer. Note that rays oriented by an angle $\theta_{1}$ from the normal are attenuated by the factor $t^{1 / \cos \theta_{1}}$ where $t$, as previsouly, denotes the transmittance of rays perpendicular to the layer. The transfer matrix attached to the layer is therefore:

$$
\mathbf{L}_{1}\left(\theta_{1}\right)=\frac{1}{T_{1}^{1 / \cos \theta_{1}}}\left(\begin{array}{cc}
T_{1}^{2 / \cos \theta_{1}} & 0 \\
0 & 1
\end{array}\right)
$$

Equation (76) becomes

$$
\left(\begin{array}{c}
J_{0}\left(\theta_{0}\right) \\
I_{0}\left(\theta_{0}\right)
\end{array}\right)=\mathbf{F}_{01}\left(\theta_{0}\right) \mathbf{L}_{1}\left(\theta_{1}\right) \mathbf{F}_{12}\left(\theta_{1}\right)\left(\begin{array}{c}
J_{2}\left(\theta_{2}\right) \\
I_{2}\left(\theta_{2}\right)
\end{array}\right)
$$

and the transfer matrix of the film is given by the matrix product $\mathbf{F}_{01}\left(\theta_{0}\right) \mathbf{L}_{1}\left(\theta_{1}\right) \mathbf{F}_{12}\left(\theta_{1}\right)$. It seems not necessary to expand the full expression of this matrix, but it is easy to verify that the front reflectance, obtained by dividing the top-right entry by the bottom-right entry, is

$$
R_{f}\left(\theta_{0}\right)=R_{01}\left(\theta_{0}\right)+\frac{T_{01}^{2}\left(\theta_{0}\right) R_{12}\left(\theta_{1}\right) T_{1}^{2 / \cos \theta_{1}}}{1-R_{12}\left(\theta_{1}\right) R_{01}\left(\theta_{0}\right) T_{1}^{2 / \cos \theta_{1}}}
$$

and that the forward transmittance, inverse of the bottom-right entry, is

$$
T_{f}\left(\theta_{0}\right)=\frac{T_{01}\left(\theta_{0}\right) T_{1}^{1 / \cos \theta_{1}} T_{12}\left(\theta_{1}\right)}{1-R_{12}\left(\theta_{1}\right) R_{01}\left(\theta_{0}\right) T_{1}^{2 / \cos \theta_{1}}}
$$

We can also verify that equations (78) and (79) are special cases of these expressions (86) and (87) when $\theta_{0}=0$, and that equations (61) and (62) of [HDI062], obtained from geometrical series, are also special cases of (86) and (87) when media 1 and 3 are air.

\subsection{Nonscattering multilayers}

The line of reasoning presented for the case of one film extends in a straightforward manner to the case of nonscattering multilayers where the layers have distinct indices. By way of illustration, the flux transfers in the case of two layers are represented in the Figure 9. 


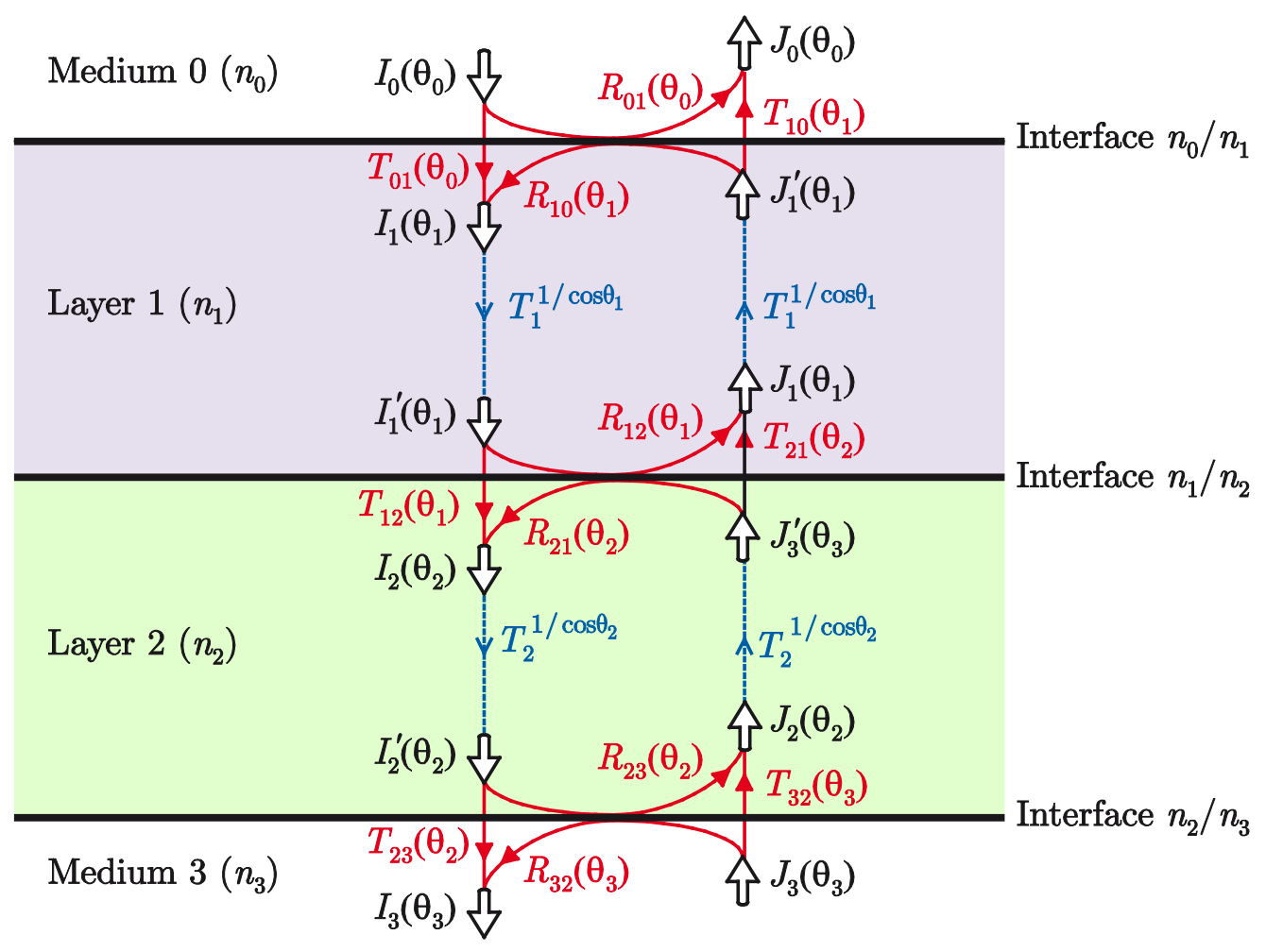

Figure 9. Flux transfers in two nonscattering layers with different indices.

The orientation of light in media $k=1,2$ and 3 are functions of the incident angle $\theta_{0}$ in medium 0

$$
\theta_{3}=\arcsin \left(n_{3} \sin \theta_{3} / n_{j}\right)
$$

If we want to consider illumination from the back side, the orientation of light in media $j=0$, 1 and 2 can be expressed as functions of the incident angle $\theta_{3}$ in medium 3 ,

$$
\theta_{j}=\arcsin \left(n_{3} \sin \theta_{3} / n_{j}\right)
$$

The matrix model directly enables writing the transfer matrix $\mathbf{M}$ of the bilayer as

$$
\mathbf{M}\left(\theta_{0}\right)=\mathbf{F}_{01}\left(\theta_{0}\right) \mathbf{L}_{t_{1}}\left(\theta_{1}\right) \mathbf{F}_{12}\left(\theta_{1}\right) \mathbf{L}_{t_{2}}\left(\theta_{2}\right) \mathbf{F}_{23}\left(\theta_{2}\right)
$$

where the matrices $\mathbf{F}_{j k}\left(\theta_{k}\right)$ are the transfer matrices attached to the interfaces defined by equation (83) and the matrices $\mathbf{L}_{t_{k}}\left(\theta_{k}\right)$ are the transfer matrices attached to the layers defined by equation ( 84$)$.

The matrix method applies with any stack of nonscattering layers, for any incident angle, provided each layer is thicker than the coherence length of light. Various examples are proposed in the next sections. The orientation of light in the different layers is automatically embodied in the transfer matrices provided they are defined and multiplied correctly. Since 
total reflections may occur, it is recommended that one adopts the alternative matrix formalism proposed in appendix.

The reflectance and transmittance expressions given above are relative to collimated fluxes. If we rather consider radiances, we must consider the changes of solid angle due to the refractions at the interfaces, which modifies the density of flux per unit solid angle. We follow the reasoning developed in HDI062, Sections 3.5 and 3.6 for one interface: the incident, reflected and transmitted radiances, respectively denoted as $L_{0}, L_{R}$, and $L_{N}$, are oriented according to the angles $\theta_{0}, \theta_{R}=\theta_{0}$, and respectively $\theta_{N}$ where $N$ denotes the labeling number of the surrounding medium at the back side. Since reflected and incident radiances fulfill equal solid angles, we have

$$
L_{R}\left(\theta_{0}\right)=R_{\text {stack }}\left(\theta_{0}\right) L_{0}\left(\theta_{0}\right)
$$

where $R_{\text {stack }}\left(\theta_{0}\right)$ is the front reflectance of the stack of nonscattering layers at the angle $\theta_{0}$.

The transmitted radiance fulfills a different solid angle in medium $N$. This introduces a factor $\left(n_{N} / n_{0}\right)^{2}$, which depends only on the refractive indices of the surrounding media 0 and $N$ :

$$
L_{N}\left(\theta_{N}\right)=\left(n_{N} / n_{0}\right)^{2} T_{\text {stack }}\left(\theta_{0}\right) L_{0}\left(\theta_{0}\right)
$$

where $T_{\text {stack }}\left(\theta_{0}\right)$ is the forward transmittance of the stack of nonscattering layers at the angle $\theta_{0}$.

When the sample is illuminated with Lambertian light from medium 0 , the reflectance $\tilde{R}_{\text {stack }}$ accounts for all the rays in the hemisphere by integrating the angular reflectance $R_{\text {stack }}\left(\theta_{0}\right)$ in a similar way as in equation (46) in [HDI062]:

$$
\tilde{R}_{\text {stack }}=\int_{\theta_{0}=0}^{\pi / 2} R_{\text {stack }}\left(\theta_{0}\right) \sin 2 \theta_{0} d \theta_{0}
$$

This integral holds for transmittance, by replacing $R_{\text {stack }}\left(\theta_{0}\right)$ with $T_{\text {stack }}\left(\theta_{0}\right)$.

\subsection{Stacks of identical nonscattering films}

Let us now consider stacks of identical films illuminated at normal incidence. We first assume that there is no optical contact between the films, i.e. a thin air slice remains between them. Since a layer of air transmits all light and does not reflect it, the corresponding transfer matrix is simply the identity matrix. The reflectance $R_{f}$ and transmittance $T_{f}$ of one film at normal incidence (including multiple reflections between their interfaces with air and transmissions through the film bulk) are given by equations (78) and (79). The corresponding transfer matrix, given by (77), may also be written 


$$
\mathbf{M}=\frac{1}{T_{f}}\left(\begin{array}{cc}
T_{f}^{2}-R_{f}^{2} & R_{f} \\
-R_{f} & 1
\end{array}\right)
$$

Since light is not scattered in the stack, it remains perpendicular to all films. Matrix $\mathbf{M}$ can therefore be attached to each of them. The transfer matrix for the stack of films is therefore

$$
\mathbf{M}_{N}=\mathbf{M}^{N}
$$

whose computation can be performed through the diagonalization of $\mathbf{M}$ [16]:

$$
\mathbf{M}=\frac{1}{T_{f}} \mathbf{E} \cdot\left(\begin{array}{cc}
v_{1} & 0 \\
0 & v_{2}
\end{array}\right) \cdot \mathbf{E}^{-1}
$$

where $v_{1}=1-(\alpha-\beta) R_{f}, v_{2}=1-(\alpha+\beta) R_{f}$ and

$$
\mathbf{E}=\left(\begin{array}{cc}
\alpha-\beta & \alpha+\beta \\
1 & 1
\end{array}\right),
$$

with

$$
\alpha=\frac{1+R_{f}^{2}-T_{f}^{2}}{2 R_{f}}
$$

and

$$
\beta=\sqrt{\alpha^{2}-1}
$$

Note that the relationship between $\alpha$ and $\beta$ [equation (99)] is similar to the one between $a$ and $b$ in the Kubelka-Munk model [equation (20)]. We thus have $(\alpha+\beta)(\alpha-\beta)=1$, and

$$
\begin{aligned}
\mathbf{M}^{N} & =\frac{1}{T_{f}^{N}} \mathbf{E} \cdot\left(\begin{array}{cc}
v_{1}^{N} & 0 \\
0 & v_{2}^{N}
\end{array}\right) \cdot \mathbf{E}^{-1} \\
& =\frac{1}{2 b T_{f}^{N}}\left(\begin{array}{cc}
(\alpha+\beta) v_{2}^{N}-(\alpha-\beta) v_{1}^{N} & v_{1}^{N}-v_{2}^{N} \\
v_{2}^{N}-v_{1}^{N} & (\alpha+\beta) v_{1}^{N}-(\alpha-\beta) v_{2}^{N}
\end{array}\right)
\end{aligned}
$$

The reflectance $R_{N}$ is given by the ratio of entries $m_{12} / m_{22}$ of matrix $\mathbf{M}_{N}$ :

$$
R_{N}=\frac{v_{1}^{N}-v_{2}^{N}}{(\alpha-\beta) v_{1}^{N}-(\alpha+\beta) v_{2}^{N}}
$$

After replacing $v_{1}$ and $v_{2}$ with their respective expressions and rearranging, equation (101) becomes: 


$$
R_{N}=\frac{1}{\alpha-\beta\left(1-\frac{2}{1-\left[\frac{1-(\alpha+\beta) R_{f}}{1-(\alpha-\beta) R_{f}}\right]^{N}}\right)} .
$$

The transmittance of the stack of films is the inverse of entry $m_{22}$ of $\mathbf{M}_{N}$

$$
T_{N}=\frac{2 b T_{f}^{N}}{(\alpha+\beta)\left[1-(\alpha-\beta) R_{f}\right]^{N}-(\alpha-\beta)\left[1-(\alpha+\beta) R_{f}\right]^{N}}
$$

As $N$ increases, the stack contains more interfaces and thus becomes more reflecting. It also contains more layers and becomes more absorbing. The reflectance varies until a limit value corresponding to the reflectance of an infinite stack, denoted as $R_{\infty}$. Since the term raised at the power $N$ in equation (102) is smaller than 1 , it tends to zero. The infinite stack reflectance is therefore

$$
R_{\infty}=\frac{1}{\alpha+\beta}=\alpha-\beta
$$

This variation is illustrated by the top-left graph in Figure 10 showing the spectral reflectances of stacks of blue acetate films $\left(n_{1}=1.54\right)$, studied in depth in Ref [17]. In this case, the reflectance increases with the number of films for all wavelengths, despite reaching rapidly a limit value in the spectral domain where the acetate is the more absorbing (570 $680 \mathrm{~nm}$ ). Outside this waveband, the reflectance increases more regularly according to the number of added film-air interfaces. In contrast, since the photons have less chance to cross the multilayer without being absorbed or back-reflected by interfaces, the transmittance decreases. It strives rapidly towards zero in the absorption waveband $570-680 \mathrm{~nm}$ and decreases more regularly and slowly outside the absorption waveband.

In order to change the relative index of the film surfaces, we can fill the interstices between films with a clear liquid, e.g. oil or water, with index denoted as $n_{2}$ (Figure 11). In this case, the interfaces in contact with the liquid are less reflecting than the front and back interfaces of the stack which are in contact with air. The global reflectance and transmittance of the stack are thus modified, as shown by comparing the top and middle graphs in Figure 10, corresponding respectively to interstices filled with air and alcohol-based liquid $\left(n_{2}=1.4\right)$. 

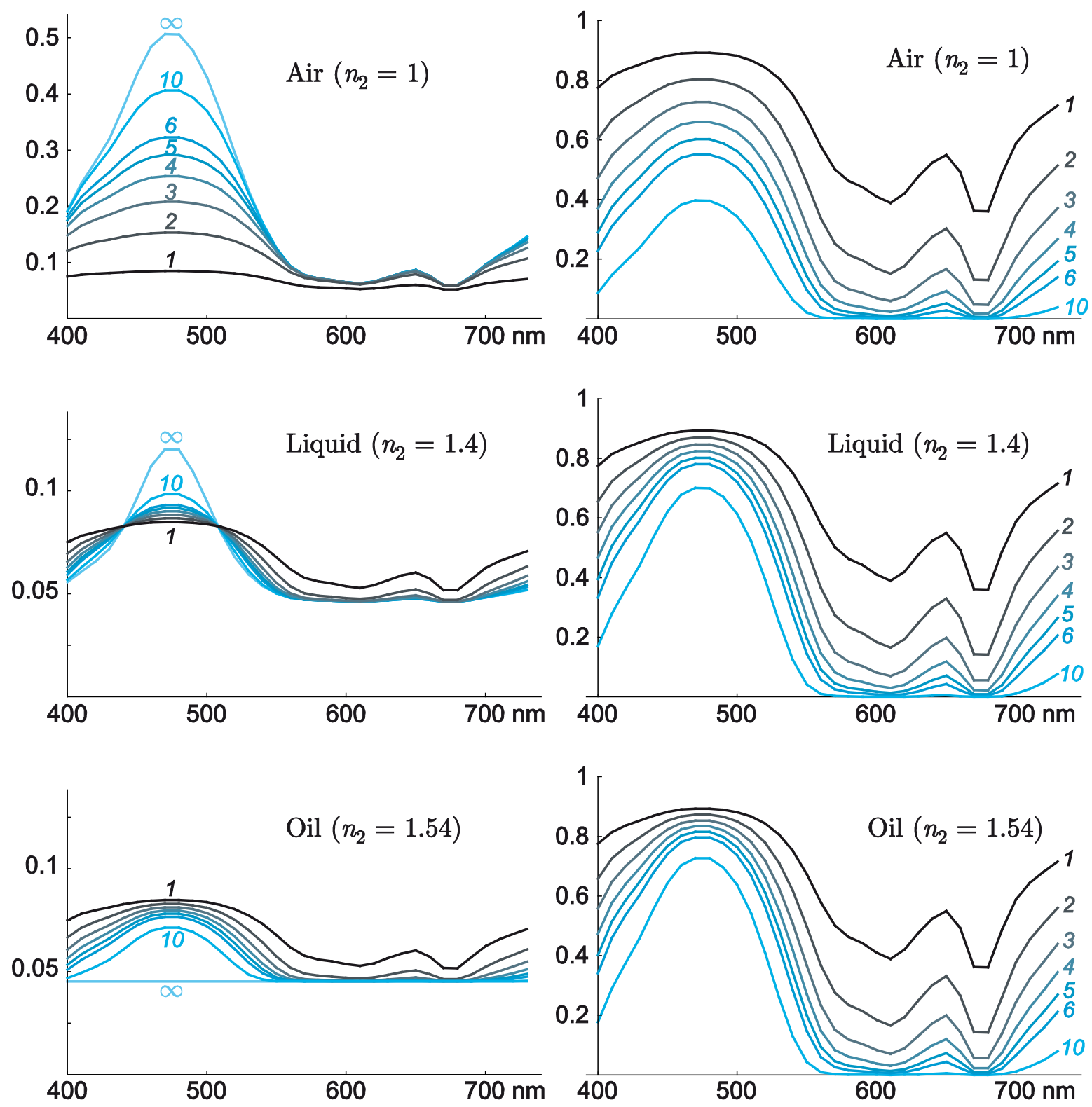

Figure 10. Predicted spectral reflectances and transmittances at normal incidence of stacks of blue acetate films $\left(1,2,3,4,5,6\right.$ and 10 films as well as, for reflectance, infinity) separated with different media (index $\left.n_{2}\right)$.

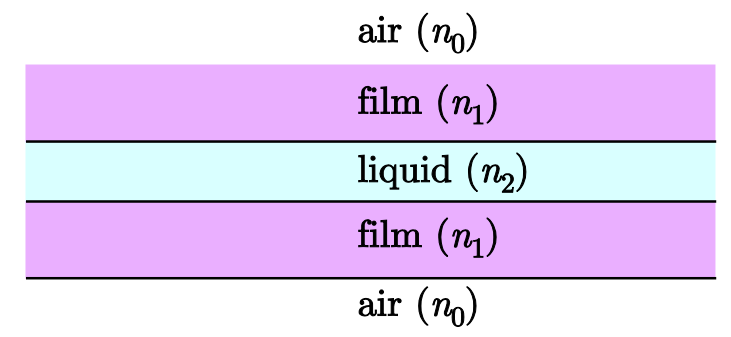

Figure 11. Stack of two acetate films pasted with liquid. 
The transfer matrices of the interfaces are denoted $\mathbf{F}_{j k}$ according to the indices of the media $j$ and $k$ being respectively at the front and back side of the interface. They are defined in equation (71). The transfer matrix of a film layer, $\mathbf{L}_{t}$, is given by equation (75). Recall that all these transfer matrices are defined for normal incidence of light. For a stack of two films, the transfer matrix is given by

$$
\mathbf{M}_{2}=\left(\mathbf{F}_{01} \cdot \mathbf{L}_{t} \cdot \mathbf{F}_{12}\right) \cdot\left(\mathbf{F}_{21} \cdot \mathbf{L}_{t} \cdot \mathbf{F}_{10}\right)
$$

and for a stack of $N$ films, it becomes:

$$
\mathbf{M}_{N}=\left(\mathbf{F}_{01} \cdot \mathbf{L}_{t} \cdot \mathbf{F}_{12}\right) \cdot\left(\mathbf{F}_{21} \cdot \mathbf{L}_{t} \cdot \mathbf{F}_{12}\right)^{N-2} \cdot\left(\mathbf{F}_{21} \cdot \mathbf{L}_{t} \cdot \mathbf{F}_{10}\right)
$$

The reflectances and transmittances of the stacks of films are deduced from the entries of the transfer matrices as indicated by equations (57). Their analytical expressions are too long to be shown here, but their numerical computation is not difficult.

It is interesting to observe in the middle graphs of Figure 10 that incrementing the number of films increases the reflectance in the waveband $440-510 \mathrm{~nm}$ where the films are less absorbing and decreases outside this domain. At $440 \mathrm{~nm}$ and $510 \mathrm{~nm}$, the reflectance does not vary because there is a perfect compensation between the gain due to back-reflection of light by the interfaces and the loss due to absorption. The transmittance follows a similar evolution when the films are separated by air, despite a slower decrease due to the higher transmittance of the interfaces.

In the special case where the liquid has the same refractive index as the films (oil with index 1.54), the interfaces in contact with it do not have optical effect any more. The transfer matrix can be written

$$
\mathbf{M}_{N}=\mathbf{F}_{01} \cdot \mathbf{L}_{t}^{N} \cdot \mathbf{F}_{10}
$$

The stack becomes equivalent to one thicker film with normal transmittance $t^{N}, N$ being the number of films. The reflectance and transmittance of this layer are similarly expressed as those of a single film, with $t^{N}$ in place of $t$. The reflectance comes only from the reflection of light by the front and back interfaces which are in contact with air. As the number of films goes to infinity, the light entering the stack is entirely absorbed and only the light reflected by the front interface is observed. The spectral reflectance is 0.04 for all wavelengths, thus yielding an achromatic color. 


\subsection{Stacks of printed films}

As an extension of the previous example, we propose to study stacks of films printed with inks, illuminated at normal incidence. Assuming that the refractive index of the films and the inks are equal, from an optical point of view, the printed film is very similar to a colored film: it is bounded by similar interfaces and the film substrate layer is replaced with two layers in optical contact with same index. The transfer matrix of the printed film, $\mathbf{L}_{p}$, is thus the product of those of the film substrate, $\mathbf{L}_{t_{s}}$, and the ink layer, $\mathbf{L}_{t_{i}}$

$$
\mathbf{L}_{p}=\mathbf{L}_{t_{s}} \cdot \mathbf{L}_{t_{i}}=\frac{1}{t_{s} t_{i}}\left(\begin{array}{cc}
1 & 0 \\
0 & t_{s}^{2} t_{i}^{2}
\end{array}\right)
$$

which indicates that the normal transmittance of the printed film is simply the product of the normal transmittances of the film and the ink layer, respectively $t_{s}$ and $t_{i}$. In theory, the printed film should have the same reflectance and transmittance on its two faces: flipping it without changing the illumination and observation conditions should not modify its visual aspect. However, optical phenomena sometimes generate a colored sheen only visible on the face with ink. Figure 12 shows the different spectral reflectances of inked and non-inked faces of a film printed with a Canon inkjet printer. The inked face displays a purplish aspect which is not observed on the other face. The higher reflectance measured on the inked face below 350 $\mathrm{nm}$ and beyond $550 \mathrm{~nm}$ is at the origin of the purplish sheen, while the opposite face has a bluish color characteristic of cyan ink deposited on a weakly reflecting support. In transmittance, the difference between front and back sides is much smaller. We can consider with inkjet prints that the relative difference between the two transmittances, generally inferior to $1 \%$, is independent of wavelength.

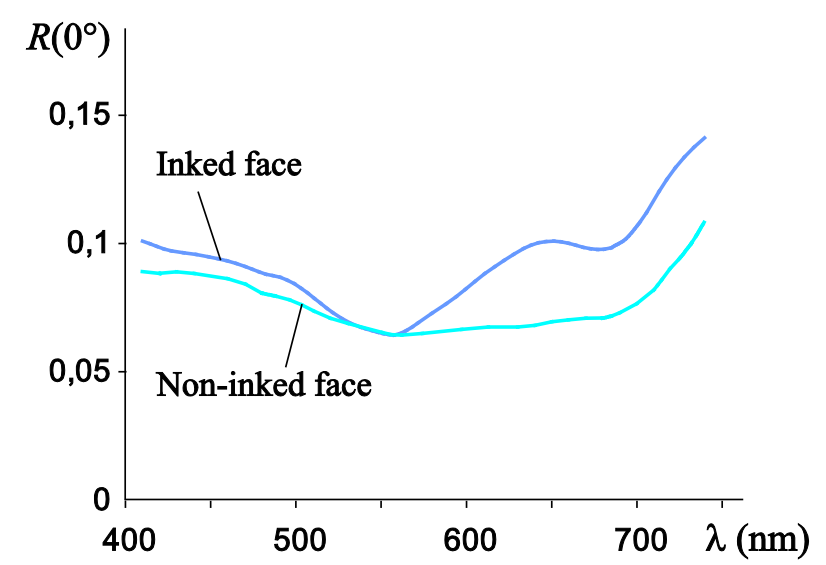

Figure 12. Spectral reflectance measured at normal incidence on the inked and non-inked faces of a film printed with cyan and yellow ink halftones at nominal surface coverages 0.57 , respectively 0.12 . 
Let us denote as $R_{f}$ and $R_{f}^{\prime}$ the reflectances of the printed film at the front side, and respectively the back side, and as $T_{f}$ and $T_{f}^{\prime}$ its front-to-back, and respectively back-to-front transmittances. These reflectances and transmittances embody the multiple reflections and transmissions of light by the interfaces, the film layer and the ink layers. The transfer matrix attached to the printed film is

$$
\mathbf{M}=\frac{1}{T_{f}}\left(\begin{array}{cc}
T_{f} T_{f}^{\prime}-R_{f} R_{f}^{\prime} & R_{f} \\
-R_{f}^{\prime} & 1
\end{array}\right)
$$

When $N$ identical films printed with the same color are superposed, matrix $\mathbf{M}$ is raised to the power $N$. We may follow similar reasoning as in Section 4.4 by diagonalizing matrix $\mathbf{M}$. Equations (96) to (103) remain valid, except the expressions for $\alpha, \beta$ and $R_{\infty}$ :

$$
\begin{gathered}
\alpha=\frac{1+R_{f} R_{f}^{\prime}-T_{f} T_{f}^{\prime}}{2 R_{f}}, \\
\beta=\sqrt{\alpha^{2}-\frac{R_{f}^{\prime}}{R_{f}}} .
\end{gathered}
$$

and

$$
R_{\infty}=(\alpha-\beta) \frac{R_{f}}{R_{f}^{\prime}}=\frac{1}{\alpha+\beta}
$$

Since each film has different reflectances on their two sides, stacks also have different reflectances on their two faces. The front and back reflectances are related according to:

$$
R_{N}^{\prime}=R_{N} \frac{R_{f}^{\prime}}{R_{f}}
$$

and the forward and backward transmittance are related according to:

$$
T_{N}^{\prime}=T_{N}\left(\frac{T_{f}^{\prime}}{T_{f}}\right)^{N}
$$

The model is not only valid with films coated with uniform ink layer but also with films printed with halftone colors [18]. This is shown in the following experiment based on CG3460 films from 3M printed in inkjet. The halftones were generated by stochastic screening according to the algorithm proposed by Ostromoukhov [19]. Four colors were selected called "green", "blue", "magenta" and "yellow" samples. They were generated by error diffusion halftoning and produced by printing cyan, magenta, yellow and green inks at the respective surfaces coverages $\{c, m, y, g\}=\{0,0,0,0.5\}$ for green, $\{0.35,0.15,0,0\}$ for 
blue, $\{0,0.70,0,0\}$ for magenta and $\{0.10,0.10,0.80,0\}$ for yellow. For each color, $R, R^{\prime}$ and $T$ were measured on one film, then the number $N$ of films was incremented and $R_{N}, R_{N}^{\prime}$ and $T_{N}$ where measured until 16 films (15 film stacks were therefore measured for each of the three geometries). In this experiment, $T_{f}^{\prime}=\gamma T_{f}$ was assumed, where $\gamma$ is a constant independent of wavelength specified in Table 2 for each type of film. Lower $\gamma$ coincides with higher scattering: the yellow ink is more scattering than the other inks.

For each color, the front reflectances, back reflectances and forward transmittances of the 15 stacks were predicted and compared to the corresponding measured spectra in terms of equivalent color distance expressed by CIELAB $\Delta \mathrm{E}_{94}$ values. For each series of 15 measurements-predictions, the average (and maximal in bracket) $\Delta \mathrm{E}_{94}$ values are given in Table 2. Figure 13 shows the front reflectance and the forward transmittance of a single film of each color (measured, green solid lines), those of stacks of 2 to 15 films (measured, black solid line; predicted, red dashed line) and the reflectance of an infinite stack of films (predicted according to formula (112), blue dotted line).

Table 2: Average and maximum $\Delta \mathrm{E}_{94}$ values obtained for the different films and geometries.

\begin{tabular}{lcccc}
\hline Film color & $\gamma$ value & $R_{N}$ & $R_{N}^{\prime}$ & $T_{N}$ \\
\hline Green & & av. $(\max )$ & av. $(\max )$ & av. $(\max )$ \\
Blue & 0.994 & $0.12(0.23)$ & $0.45(0.64)$ & $0.49(0.96)$ \\
Magenta & 0.993 & $0.24(0.30)$ & $0.50(0.63)$ & $0.35(0.74)$ \\
Yellow & 0.993 & $0.39(0.43)$ & $0.20(0.24)$ & $1.21(1.68)$ \\
\hline
\end{tabular}

For the green and blue colors, the prediction accuracy is excellent in both reflection and transmission modes. For the other two colors, the accuracy is good in reflectance mode and poorer in transmission mode. This is certainly due to scattering: in this experiment, the yellow ink was more scattering than the other inks. By looking at far objects through different films, blurring was more pronounced with films with much yellow. This is consistent with the fact that the $\gamma$ value is lower for the yellow films. 

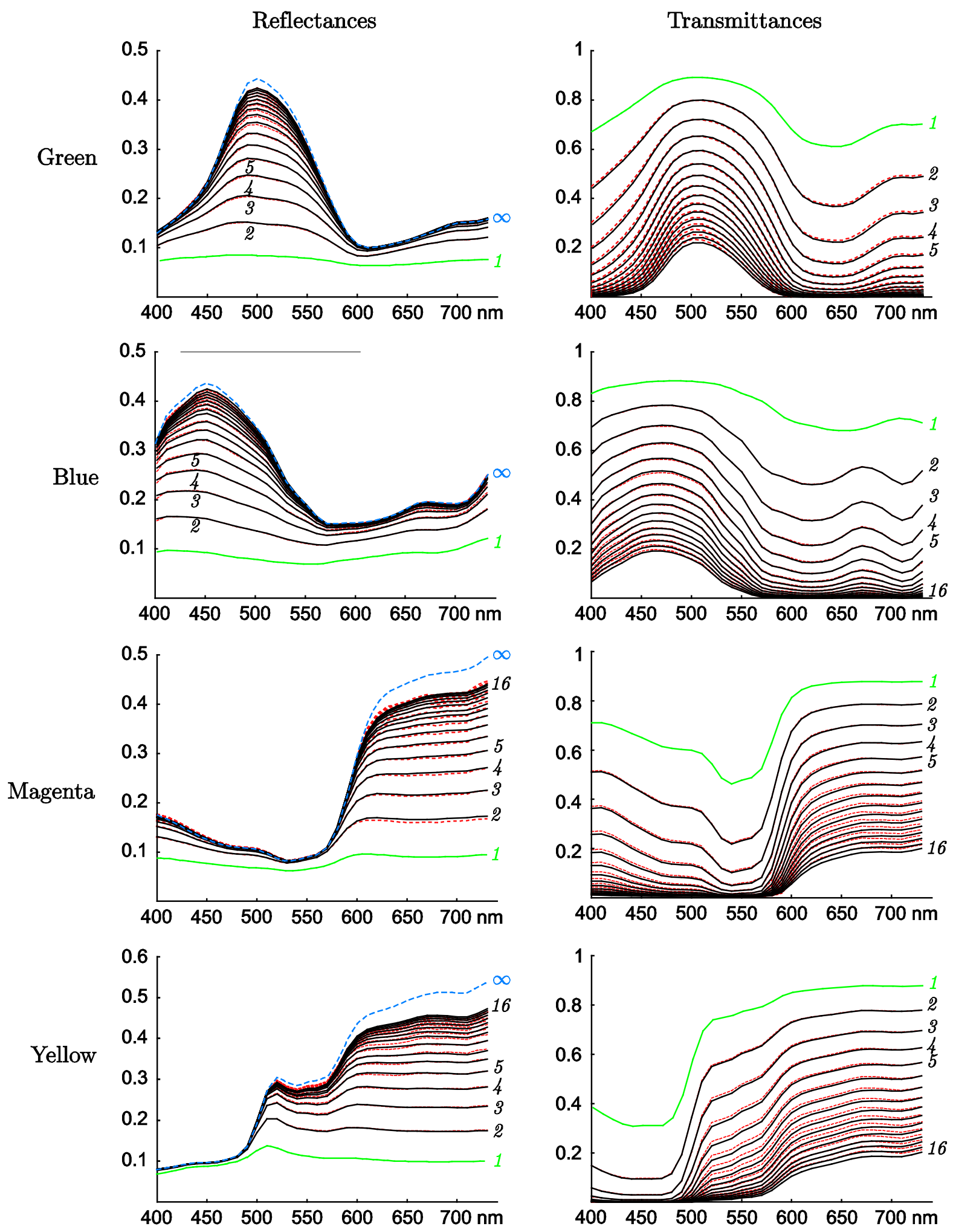

Figure 13. Front reflectances (left) and forward transmittances (right) at normal incidence of single film (measured, green lines) and of stacks of 2 to 16 green films (measured, solid black lines, and predicted, red dashed lines). The numbers at the right of the curves indicate the number of films in the stack and the symbol $\infty$ designates the predicted infinite stack reflectance (dashed blue line). 


\subsection{Stacks of printed films on top of a specular reflector}

Mirrors are also nonscattering reflectors and can be treated, despite zero transmittance, by the matrix model. The transfer matrix attached to a mirror with reflectance at normal incidence $R_{m}$ is simply defined as

$$
\mathbf{M}_{m}=\left(\begin{array}{cc}
1 & 0 \\
R_{m} & 0
\end{array}\right) .
$$

When nonscattering elements, e.g. films, are placed on it, only the front reflectance can be defined, the back reflectance and the transmittance making no sense in this case.

Let us place a stack of $N$ identical printed films in front of the mirror. As previously, we denote as $R_{f}, R_{f}^{\prime}, T_{f}$, and $T_{f}^{\prime}$ the front reflectance, back reflectance, forward transmittance and backward transmittance of one film at normal incidence. The transfer matrix attached to the specimen is

$$
\begin{aligned}
\mathbf{M} & =\left\{m_{i j}\right\}=\frac{1}{T_{f}^{N}}\left(\begin{array}{cc}
T_{f} T_{f}^{\prime}-R_{f} R_{f}^{\prime} & R_{f} \\
-R_{f}^{\prime} & 1
\end{array}\right)^{N} \cdot\left(\begin{array}{cc}
1 & 0 \\
R_{m} & 0
\end{array}\right) . \\
& =\frac{1}{T_{N}}\left(\begin{array}{cc}
0 & R_{N}+\left(T_{N} T_{N}^{\prime}-R_{N} R_{N}^{\prime}\right. \\
0 & 1-R_{N}^{\prime} R_{m}
\end{array}\right) R_{m}
\end{aligned} .
$$

where $R_{N}, R_{N}^{\prime}, T_{N}$ and $T_{N}^{\prime}$ denote the front reflectance, back reflectance, forward transmittance and backward transmittance of the stack of $N$ films at normal incidence, respectively given by Eqs. (102), (113), (103) and (114), with $a$ and $b$ given by equations (110) and (111).

Finally, the reflectance of the stack with mirror, ratio of entry $m_{12}$ to entry $m_{22}$, is

$$
R_{m, N}=R_{N}+\frac{T_{N} T_{N}^{\prime} R_{m}}{1-R_{N}^{\prime} R_{m}},
$$

valid for all wavelengths and for any $N \geq 0$.

Measured spectral reflectances $R_{m, N}(\lambda)$ are plotted in Figure 14 for stacks of the blue and green printed films previously studied, respectively placed in front of a red reflector (copper mirror covered by a film coated with red ink) and a magenta reflector (achromatic mirror covered by a film coated with magenta ink). In both cases, the number of films was incremented from 1 to 13 . The predictions given by Eq. (117) satisfyingly match the measurements: the average $\Delta \mathrm{E} 94$ value assessing the deviations between predicted and 
measured spectra was 0.42 units (maximum 0.66 units) for the green films on the magenta background, and 0.64 units (maximum 0.75 units) for the blue films on the red background.

Through these examples, we observe that as the number of films in front of the mirror increases, the spectral reflectance of the samples varies differently with the wavelength, or more precisely according to the relative values of the mirror reflectance and the infinite stack reflectance. Hence, $R_{m, N}$ either increases or decreases and may even be constant for wavelengths where the spectral reflectances of background and infinite stack meet.
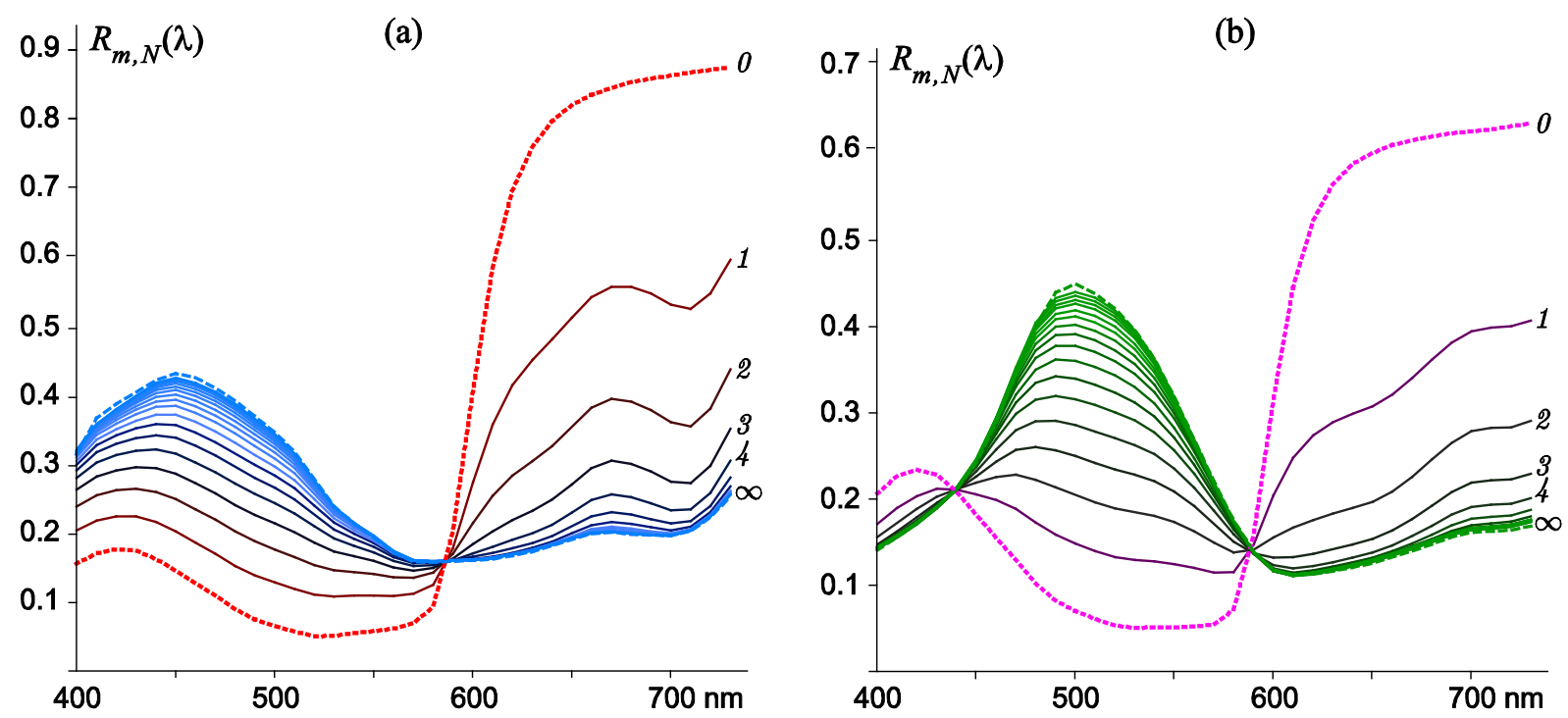

Figure 14. Spectral reflectances of (a) blue films in front of a red reflector and (b) green films in front of a magenta reflector. The numbers denote the numbers of films and line colors roughly reproduce the colors associated to the plotted spectra. Spectral reflectances of the mirror alone (label 0) and of an infinite stack of films $($ label $\infty)$ are in dotted and dashed lines respectively.

\section{STACKS OF DIFFUSING AND NONSCATTERING LAYERS}

The two-flux models presented in the previous sections for strongly diffusing layers and for non-scattering layers can be extended to mixed stacks of strongly diffusing and nonscattering layers. The transfer matrices attached to the diffusing layers are similarly defined as in equation (52). Those attached to the nonscattering layers and flat interfaces, however, must be adapted to the angular distribution of the light they receive. We saw in Section 4 that the trajectories and the attenuation of collimated light within a nonscattering multilayer are fully determined by the incident angle. Hence, every sequence of consecutive nonscattering layers and flat interfaces in the multilayer is considered as one nonscattering component. The angular transfer matrix model developed in Section 3 will help to get the analytical 
expressions for their front reflectance $R(\theta)$, back reflectance $R^{\prime}(\theta)$, forward transmittance $T(\theta)$ and backward transmittance $T^{\prime}(\theta)$. When the incident light is diffuse, we integrate these angular functions with respect to the angular distribution of light, which is in practice either collimated when it comes from a directional light source or Lambertian when it comes from a Lambertian light source or a diffusing layer. In this latter case, the angular function $X(\theta)$ is integrated over the hemisphere, thus yielding an integral similar to the one of equation (93):

$$
\tilde{X}=\int_{\theta=0}^{\pi / 2} X(\theta) \sin 2 \theta d \theta
$$

This is the case when the nonscattering component is located between two diffusing layers (Figure 15.a). The transfer matrix is therefore

$$
\frac{1}{\tilde{T}}\left(\begin{array}{cc}
\tilde{T} \tilde{T}^{\prime}-\tilde{R} \tilde{R}^{\prime} & \tilde{R} \\
-\tilde{R}^{\prime} & 1
\end{array}\right)
$$

where symbol $\sim$ denotes the integration defined by equation (118).

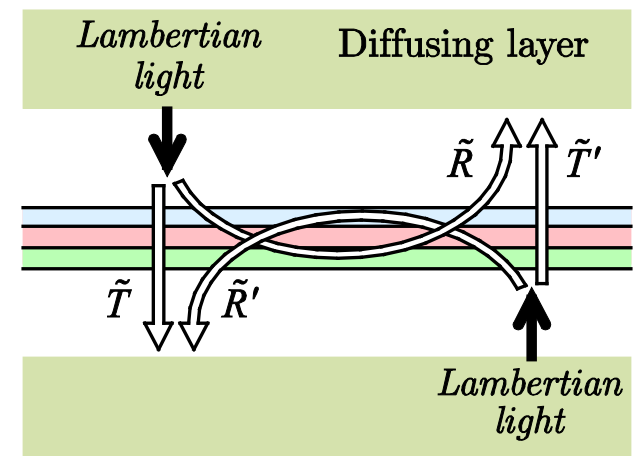

(a)

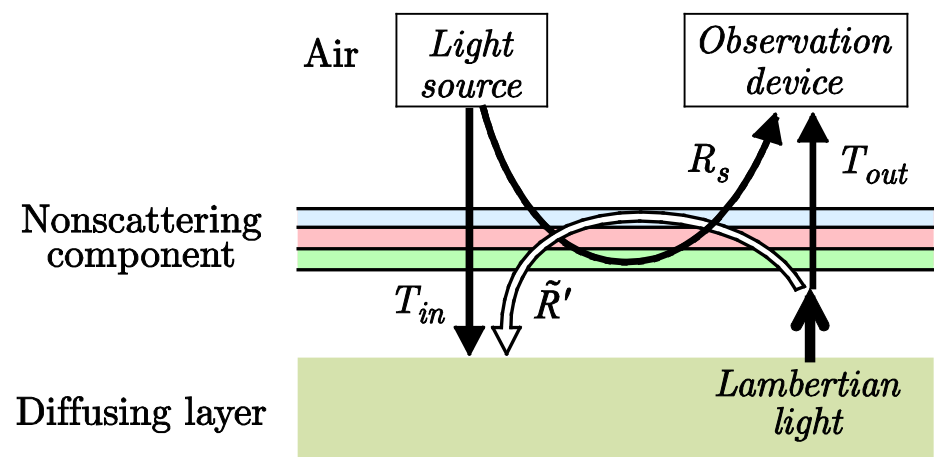

(b)

Figure 15. Flux transfers through a nonscattering element located (a) between two diffusing layers, (b) at the front edge position.

When the nonscattering component is in an external position, for example at the front side, its global front reflectance, back reflectance, forward transmittance and backward transmittance, respectively denoted $R_{S}, R_{i}, T_{i n}$ and $T_{\text {out }}$, account for the angular geometry of the light source and detection system (see Figure 15.b). Their expressions in terms of the angular functions $R(\theta), R^{\prime}(\theta), T(\theta)$ and $T^{\prime}(\theta)$, are given in Table 3 for typical configurations: collimated (e.g. $0^{\circ}$ or $45^{\circ}$ ) or Lambertian incident light and observation over the hemisphere or in one direction. The forward transmittance depends only on the illumination geometry and the back transmittance only on the observation geometry. If light is collected over the hemisphere, and 
since the light coming from the diffusing layer is also Lambertian, the back transmittance is $\tilde{T}$. In the case of a directional observation, only the radiance directed toward the observer is considered. It corresponds to a fraction $1 / \pi$ of the Lambertian irradiance issued from the diffusing layer, attenuated by the angular reflectance of the nonscattering component, and divided by the term $n^{2}$ in order to take into account the change of solid angle due to the refractions, where $n$ is the refractive index of the diffusing medium (see equation (92), the index of air being 1 in the present case). The front reflectance $R_{S}$ depends on both illumination and observation geometries. When the incident light is collimated, it is the angular reflectance $R(\theta)$ evaluated at the incidence angle provided the reflected light is collected by the detection system; otherwise it is zero. When the incident light is Lambertian, $R_{s}$ is the diffuse reflectance $\tilde{R}$ if all the reflected light is collected by an integrating sphere; it is the angular reflectance $R(\theta)$ evaluated at the observation angle if the detection device is collimated, knowing that only a fraction $1 / \pi$ of the Lambertian irradiance is in the concerned direction. Lastly, the back reflectance $R_{i}$ is independent of the measuring geometry: it accounts for the reflection of the Lambertian light issued from the diffusing layer:

$$
R_{i}=\tilde{R}^{\prime}=\int_{\theta=0}^{\pi / 2} R^{\prime}(\theta) \sin 2 \theta d \theta
$$

The transfer matrix attached to the nonscattering component is

$$
\frac{1}{T_{\text {in }}}\left(\begin{array}{cc}
T_{\text {in }} T_{\text {out }}-R_{s} R_{i} & R_{s} \\
-R_{i} & 1
\end{array}\right)
$$

Table 3: Expressions for $R_{S}, T_{i n}$, and $T_{\text {out }}$ according to the illumination and observation geometry

\begin{tabular}{lccc}
\hline & $R_{S}$ & $T_{\text {in }}$ & $T_{\text {out }}$ \\
\hline $\begin{array}{l}\text { Collimated incident light at angle } \\
\alpha \text { and observation over the } \\
\text { hemisphere }\end{array}$ & $R(\alpha)$ & $T(\alpha)$ & $\tilde{T}^{\prime}$ \\
\hline $\begin{array}{l}\text { Collimated incident light at angle } \\
\alpha \text { and observation in one direction } \\
\psi\end{array}$ & $\begin{array}{l}R(\alpha) \text { if } \alpha=\psi \\
0 \text { otherwise }\end{array}$ & $T(\alpha)$ & $T^{\prime}(\psi) / n^{2}$ \\
$\begin{array}{l}\text { Lambertian incident light and } \\
\text { observation over the hemisphere }\end{array}$ & $\tilde{R}$ & $\tilde{T}$ & $\tilde{T}^{\prime}$ \\
\hline $\begin{array}{l}\text { Lambertian incident light and } \\
\text { observation in one direction } \psi\end{array}$ & $R(\psi) / \pi$ & $\tilde{T}$ & $T^{\prime}(\psi) / \pi n^{2}$ \\
\hline
\end{tabular}


Once all the transfer matrices attached to the nonscattering components (i.e. the sequences of nonscattering layers and interfaces) are determined, they can be multiplied with those attached to the diffusing layers. For example, a nonscattering component on top of a symmetric diffusing layer with reflectance $\rho$ and transmittance $\tau$ has the transfer matrix given by

$$
\begin{aligned}
\mathbf{Q} & =\frac{1}{T_{\text {in }}}\left(\begin{array}{cc}
T_{\text {in }} T_{\text {out }}-R_{S} R_{i} & R_{s} \\
-R_{i} & 1
\end{array}\right) \cdot \frac{1}{\tau}\left(\begin{array}{cc}
\tau^{2}-\rho^{2} & \rho \\
-\rho & 1
\end{array}\right) \\
& =\frac{1}{T_{\text {in }} \tau}\left(\begin{array}{cc}
-R_{s} \rho+\left(T_{\text {in }} T_{\text {out }}-R_{S} R_{i}\right)\left(\tau^{2}-\rho^{2}\right) & R_{S}+\left(T_{\text {in }} T_{\text {out }}-R_{S} R_{i}\right) \rho \\
-\rho-R_{i}\left(\tau^{2}-\rho^{2}\right) & 1-R_{i} \rho
\end{array}\right)
\end{aligned}
$$

We can deduce from it the front reflectance (ratio of entries $q_{12} / q_{22}$ )

$$
R_{\text {sample }}=R_{s}+\frac{T_{i n} T_{\text {out }} \rho}{1-R_{i} \rho}
$$

or the back transmittance ( $\operatorname{det} \mathbf{Q} / q_{22}$ ), corresponding to the same observation conditions as the front reflectance, with the light coming from beside the diffusing layer:

$$
T_{\text {sample }}^{\prime}=\frac{T_{\text {out }} \tau}{1-R_{i} \rho}
$$

We may imagine specimens consisting of several alternations of diffusing and nonscattering components. The examples which are proposed in the next sections will be based on this configuration, the simplest being a nonscattering component is on top of a diffusing layer.

\subsection{Paper coated with gelatin (Extended Williams-Clapper model)}

In 1953, Williams and Clapper proposed a prediction model for the spectral reflectance of glossy photographs, made of paper coated with photochromic gelatin [20]. The original Williams-Clapper reflectance formula, derived from a description of the multiple reflections between the paper and the surface yielding a geometrical series, is based on the $45^{\circ}: 0^{\circ}$ bidirectional geometry. A formula based on the diffuse-directional geometry was later derived by Shore and Spoonhower [21] and has been recently extended to the transmittance [15]. All these formulas can be obtained with the matrix method, for which the interest is rather limited in such a simple case but offers a good example of application. The interest of the method will be more striking when increasing the number of layers.

The photographic paper is composed of the air-gelatin interface, the gelatin layer with spectral normal transmittance $T_{g}(\lambda)$, and the paper support with spectral reflectance $\rho(\lambda)$ and 
spectral transmittance $\tau(\lambda)$. Paper and gelatin are assumed to have the same refractive index $n$. Since, they are in optical contact, the interface between them has no optical effect.

The air-gelatin interface and the gelatin layer are two consecutive nonscattering elements located at the front position. They are considered as one nonscattering component whose transfer matrix is similar to equation (121). The transfer matrix attached to the photographic paper is therefore similar to equation (122) and its reflectance has an expression similar to equation (123). In this reflectance expression, $R_{s}, T_{i n}, T_{\text {out }}$ and $R_{i}$ are functions of the normal transmittance $T_{g}(\lambda)$ and the index $n_{g}$ of the gelatin and depend on the measuring geometry. They are obtained in two steps.

The first step is to compute the angular transfer matrix $\mathbf{G}\left(\theta_{0}\right)$ of this nonscattering component. It is the product of the angular transfer matrix attached to the air-gelatin interface, given by equation (82) with labels $j=0$ for air and $k=1$ for the gelatin, and the angular transfer matrix attached to the gelatin layer given by equation (84):

$$
\begin{aligned}
\mathbf{G}\left(\theta_{0}\right) & =\mathbf{F}_{01}\left(\theta_{0}\right) \mathbf{L}_{g}\left(\theta_{1}\right) \\
& =\frac{1}{T_{01}\left(\theta_{0}\right)\left[T_{g}(\lambda)\right]^{1 / \cos \theta_{1}}}\left(\begin{array}{cc}
{\left[T_{01}\left(\theta_{0}\right) T_{10}\left(\theta_{1}\right)-R_{01}\left(\theta_{0}\right) R_{10}\left(\theta_{1}\right)\right]\left[T_{g}(\lambda)\right]^{2 / \cos \theta_{1}}} & R_{01}\left(\theta_{0}\right) \\
-R_{10}\left(\theta_{1}\right)\left[T_{g}(\lambda)\right]^{2 / \cos \theta_{1}} & 1
\end{array}\right)
\end{aligned}
$$

where $\theta_{0}$ denotes the orientation of light in air, $\theta_{1}=\arcsin \left(\sin \theta_{0} / n\right)$ the corresponding orientation in the gelatin and $R_{01}\left(\theta_{0}\right)$ the Fresnel angular reflectance of the interface at the air side.

We deduce from $\mathbf{G}\left(\theta_{0}\right)$ the front angular reflectance, $R_{01}\left(\theta_{0}\right)$, the back angular reflectance, $R_{10}\left(\theta_{1}\right)\left[T_{g}(\lambda)\right]^{2 / \cos \theta_{1}}$, and the upward and downward transmittances, $T_{01}\left(\theta_{0}\right)\left[T_{g}(\lambda)\right]^{1 / \cos \theta_{1}}$. The exponents can be expressed as functions of $\theta_{0}$ thanks to the following transformation:

$$
\cos \theta_{1}=\cos \left(\arcsin \left(\sin \theta_{0} / n\right)\right)=\sqrt{1-\left(\sin \theta_{0} / n\right)^{2}}
$$

In a second step, we consider the orientations of light in the nonscattering component, assuming a $45^{\circ}: 0^{\circ}$ measuring geometry as Williams and Clapper in their original paper. According to the formulas given in Table 3, we obtain:

$$
\begin{gathered}
R_{s}=0 \\
T_{\text {in }}(\lambda)=T_{01}\left(45^{\circ}\right) T_{g}^{\beta}(\lambda)
\end{gathered}
$$


with

$$
\begin{gathered}
\beta=\frac{2 n_{g}}{\sqrt{4 n_{g}^{2}-1}}, \\
T_{\text {out }}(\lambda)=\frac{T_{10}(0) T_{g}(\lambda)}{\pi n_{g}^{2}},
\end{gathered}
$$

and

$$
R_{i}(\lambda)=\int_{0}^{\pi / 2} R_{10}\left(\theta_{1}\right)\left[T_{g}(\lambda)\right]^{2 / \cos \theta_{1}} \sin 2 \theta_{1} d \theta_{1} \approx \tilde{R}_{10} \frac{\exp \left(T_{g}^{\gamma}(\lambda)\right)-1}{\exp (1)-1}
$$

where $\tilde{R}_{10}$ is the diffuse reflectance of the surface at the gelatin-side (also denoted as $r_{10}$ in [HDI062], Section 3.6) and $\gamma$ a coefficient minimizing the difference between the integral and the approximation function for the considered refractive index $\left(\gamma=2.91\right.$ for $n_{g}=1.53$, see Table 4).

Finally, the reflectance formula (123), multiplied by $\pi$, gives the spectral reflectance factor of the photographic paper which would be measured with a commercial instrument calibrated with respect to the reflectance of a perfectly white diffuser (equal to $1 / \pi$ ):

$$
\hat{R}_{W C}^{45: 0}(\lambda)=\frac{R_{W C}^{45: 0}(\lambda)}{1 / \pi}=\frac{1}{n_{g}^{2}} \cdot \frac{T_{01}\left(45^{\circ}\right) T_{10}\left(0^{\circ}\right) T_{g}^{1+\beta}(\lambda) \rho(\lambda)}{1-r_{10}\left(\frac{\exp \left[T_{g}^{\gamma}(\lambda)\right]^{-1}}{e-1}\right) \rho(\lambda)}
$$

Since the spectral reflectance $\rho(\lambda)$ of the paper substrate cannot be measured directly due to the presence of the paper-air interface, we want to deduce it from measurement. We thus take the paper in an area where the gelatin is uncolored (normal transmittance 1) or, if available, the paper without gelatin provided its surface is flat. The reflectance factor given by equation (127) thus becomes

$$
\hat{R}_{p}(\lambda)=\frac{1}{n_{g}^{2}} \frac{T_{01}\left(45^{\circ}\right) T_{10}\left(0^{\circ}\right) \rho(\lambda)}{1-r_{10} \rho(\lambda)}
$$

Assuming $n$ is known, we can compute the Fresnel functions and only the background reflectance $\rho$ remains unknown. We obtain it by reversing the equation (128):

$$
\rho(\lambda)=\frac{n^{2} \hat{R}_{p}(\lambda)}{T_{01}\left(45^{\circ}\right) T_{10}\left(0^{\circ}\right)+n_{g}^{2} r_{10} \hat{R}_{p}(\lambda)}
$$

In the case of a d: $0^{\circ}$ geometry, according to the formulas of Table 3, we obtain: 


$$
\begin{gathered}
R_{s}=\frac{R_{01}\left(0^{\circ}\right)}{\pi}, \\
T_{\text {in }}(\lambda)=\int_{0}^{\pi / 2} T_{01}\left(\theta_{0}\right)\left[T_{g}(\lambda)\right]^{1 / \sqrt{1-\left(\sin \theta_{0} / n\right)^{2}}} \sin 2 \theta_{0} d \theta_{0} \approx \tilde{T}_{01} T_{g}^{\mu}(\lambda)
\end{gathered}
$$

where $\tilde{T}_{01}$ is the diffuse transmittance of the surface from air to gelatin (also denoted as $t_{10}$ in [HDI062], Section 4.6) and $\mu$ is again a coefficient minimizing the difference between the integral and the approximation function for a given refractive index $\left(\mu=1.13\right.$ for $n_{g}=1.53$, see Table 4), and

$$
T_{\text {out }}(\lambda)=\frac{T_{10}\left(0^{\circ}\right) T_{g}(\lambda)}{\pi n_{g}^{2}}
$$

The spectral reflectance factor in respect to the perfect diffuser is [21]

$$
\hat{R}_{W C}^{d: 0^{\circ}}(\lambda)=R_{01}\left(0^{\circ}\right)+\frac{1}{n_{g}^{2}} \cdot \frac{\tilde{T}_{01} T_{10}\left(0^{\circ}\right) T_{g}^{1+\mu}(\lambda) \rho(\lambda)}{1-\tilde{R}_{10}\left(\frac{\exp \left[T_{g}^{\gamma}(\lambda)\right]-1}{e-1}\right) \rho(\lambda)}
$$

Equation (124) gives the transmittance of the photographic paper observed in the same way as in reflectance and illuminated from behind. For a d: $0^{\circ}$ geometry, the spectral transmittance factor is

$$
\hat{T}_{W C}^{d: 0^{\circ}}(\lambda)=\pi \frac{T_{\text {out }}(\lambda) \tau(\lambda)}{1-\tilde{R}^{\prime}(\lambda) \rho(\lambda)}=\frac{1}{n_{g}^{2}} \cdot \frac{T_{10}\left(0^{\circ}\right) T_{g}(\lambda) \tau(\lambda)}{1-\tilde{R}_{10} \rho(\lambda)}
$$

This method can be easily adapted to different measuring geometries. It automatically takes into account the flux transfers between the diffusing layer and the nonscattering component by respecting the orientation-dependent attenuation of each ray in the nonscattering layer.

Table 4. Values for parameters $\gamma$ and $\mu$ as a function of the refractive index

\begin{tabular}{ccc}
\hline$n_{\sigma}$ & $\gamma$ & $\mu$ \\
\hline 1.45 & 3.03 & 1.15 \\
1.47 & 2.99 & 1.14 \\
1.50 & 2.95 & 1.13 \\
1.53 & 2.91 & 1.13 \\
1.55 & 2.88 & 1.12 \\
\hline
\end{tabular}




\subsection{Diffusing support protected with colored films}

After the photographic paper, a book cover protected by a plastic film is a second example where a nonscattering component is on top of a diffusing background. Since the film and the cover are generally not in optical contact, there is an interstice between them filled by air. We may generalize this example by considering several films identical to each other. The structure of the specimens is represented in Figure 16 for two films. Since there is a nonscattering component on top of a diffusing layer, the reflectance and transmittance have similar expressions as (123), and respectively (124). We just have to compute the parameters $R_{s}, T_{i n}, T_{\text {out }}$, and $R_{i}$ in terms of the different refractive indices, the spectral reflectance $\rho(\lambda)$ and transmittance $\tau(\lambda)$ of the diffusing layer, and the spectral normal transmittance $T_{1}(\lambda)$ of each film deduced using equation (66) of [HDI062] from the measured spectral transmittance $T_{\text {film }}(\lambda)$ of the film:

$$
T_{1}(\lambda)=\frac{\sqrt{64 n_{1}^{4}+\left(1-n_{1}^{2}\right)^{4} T_{\text {film }}^{2}(\lambda)}-8 n_{1}^{2}}{\left(1-n_{1}\right)^{4} T_{\text {film }}(\lambda)}
$$

where $n_{1}$ is the refractive index of the film.

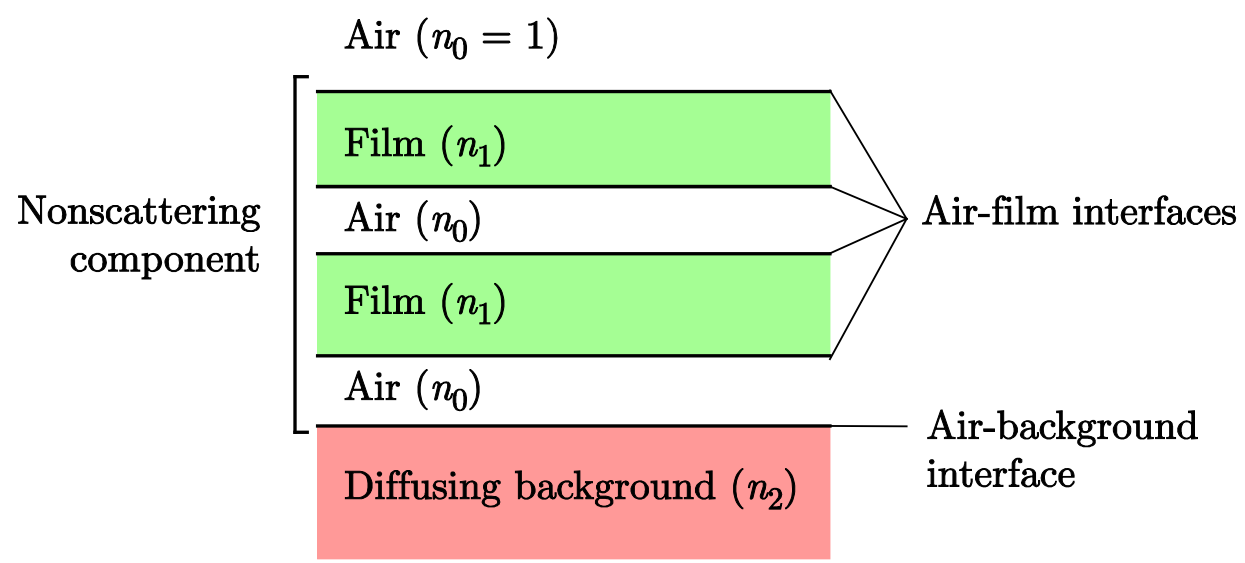

Figure 16. Nonscattering films on top of a diffusing background.

Regarding the nonscattering component, we can use the matrix model to determine its angular reflectances and transmittances, which will then be integrated with respect to the measuring geometry. We denote as $\theta_{0}$ the incident angle in air, $\theta_{j}=\arcsin \left(\sin \theta_{0} / n_{j}\right)$ the subsequent angles in the media of index $n_{j}$. The angular transfer matrix attached to it is the product of the transfer matrices attached to the different components, i.e. the interfaces and the layers, respectively, defined by equations (82) and (84). The air layers are optically neutral and the 
transfer matrices attached to them are unit matrices, and therefore omitted in the calculation. For $N$ films, the angular transfer matrix $\mathbf{M}_{N \text { films }}\left(\theta_{0}\right)$ of the nonscattering component is given by

$$
\mathbf{M}_{N \text { films }}\left(\theta_{0}\right)=\left[\mathbf{F}_{01}\left(\theta_{0}\right) \mathbf{L}_{1}\left(\theta_{1}\right) \mathbf{F}_{10}\left(\theta_{1}\right)\right]^{N} \mathbf{F}_{01}\left(\theta_{0}\right)
$$

From this angular transfer matrix, using equations (57), we obtain analytical expressions for the front reflectance $R_{N}\left(\theta_{0}\right)$, back reflectance $R_{N}^{\prime}\left(\theta_{2}\right)$, downward transmittance $T_{N}\left(\theta_{0}\right)$ and upward transmittance $T_{N}^{\prime}\left(\theta_{2}\right)$. It would take too much place to show them here, but there is no difficulty obtaining them using symbolic or numerical computation software.

Then we consider the orientations of light in the nonscattering component with respect to the measuring geometry. For the $\mathrm{d}: 0^{\circ}$ geometry that we have selected, the formulas of Table 3 give

$$
\begin{aligned}
& R_{s}=R_{N}\left(0^{\circ}\right) / \pi \\
& T_{\text {in }}=\tilde{T}_{N} \\
& T_{\text {out }}=\frac{T_{N}^{\prime}\left(0^{\circ}\right)}{\pi n_{2}^{2}} \\
& R_{i}=\tilde{R}_{N}^{\prime}
\end{aligned}
$$

where symbol $\sim$ has the same meaning as in equation (118). Note that since these four terms embody the film's normal transmittance $T_{1}(\lambda)$, all of them depend of wavelength.

In order to obtain the spectral reflectance $\rho(\lambda)$ and transmittance $\tau(\lambda)$ of the diffusing layer, we measure the spectral reflectance and transmittance factors of the diffusing support with an instrument base on the $\mathrm{d}: 0^{\circ}$ geometry. Their respective expressions are, for each wavelength,

$$
\hat{R}_{p}=R_{02}\left(0^{\circ}\right)+\frac{1}{n_{2}^{2}} \cdot \frac{\tilde{T}_{02} T_{02}\left(0^{\circ}\right) \rho}{1-\tilde{R}_{20} \rho}
$$

and

$$
\hat{T}_{p}=\frac{1}{n_{2}^{2}} \cdot \frac{T_{02}\left(0^{\circ}\right) \tau}{1-\tilde{R}_{20} \rho}
$$

where $R_{02}(0)=1-T_{02}(0)=\left(n_{2}-1\right)^{2} /\left(n_{2}+1\right)^{2}$ is the Fresnel reflectance of the interface at normal incidence, $\tilde{R}_{20}$ is its diffuse reflectance of the interface at the paper side [see HDI062, equation (46)] and $\tilde{T}_{02}=t_{02}=n_{2}^{2}\left(1-\tilde{R}_{20}\right)$ is its transmittance for Lambertian light coming from air. Since these terms, independent of wavelength, can be computed as soon as $n_{2}$ is 
known, we can deduce $\rho(\lambda)$ and $\tau(\lambda)$ from equations (135) and (136). After some rearrangements, we get:

$$
\rho(\lambda)=\frac{\hat{R}_{p}(\lambda)-R_{02}\left(0^{\circ}\right)}{\tilde{R}_{20} \hat{R}_{p}(\lambda)+1-R_{02}\left(0^{\circ}\right)}
$$

and

$$
\tau(\lambda)=\frac{n_{2}^{2} \hat{T}_{p}(\lambda)}{1-R_{02}\left(0^{\circ}\right)} \cdot\left(\frac{1-R_{02}\left(0^{\circ}\right)+\tilde{R}_{20} R_{02}\left(0^{\circ}\right)}{1-R_{02}\left(0^{\circ}\right)+\tilde{R}_{20} \hat{R}_{p}(\lambda)}\right)
$$

Finally, the spectral reflectance and the transmittance of the specimen have similar expressions as equations (123) and (124) respectively. By multiplying them by $\pi$, we obtain the following expressions for the reflectance and transmittance factors, for each wavelength:

$$
\hat{R}^{d: 0^{\circ}}=R_{N \text { films }}\left(0^{\circ}\right)+\frac{1}{n_{2}^{2}} \cdot \frac{\tilde{T}_{N} T_{N}^{\prime}\left(0^{\circ}\right) \rho}{1-\tilde{R}_{N}^{\prime} \rho}
$$

and

$$
\hat{T}^{d: 0}=\frac{1}{n_{2}^{2}} \cdot \frac{T_{N}^{\prime}\left(0^{\circ}\right) \tau}{1-\tilde{R}_{N}^{\prime} \rho}
$$

Be aware of that all terms in these equations (139) and (140), except $n_{2}$, depend on wavelength.

As a first application of the model, we selected glossy papers printed in inkjet with various fulltone colors (cyan, magenta, red, green, blue and black) and halftone colors (cyan and magenta inks at 50\%). Figure 17 shows the normal transmittance of the blue acetate film as well as the changing of reflectance spectrum due to the presence of the film for the fulltone magenta sample. The reflectance and the transmittance of each paper covered by one film were predicted by the model above, with $k=1$ in this case. The differences between predictions and measurement were calculated in terms of color distances by computing the CIELAB $\Delta \mathrm{E}_{94}$ values in respect to the D65 standard illuminant. In reflectance mode, we obtained an average $\Delta \mathrm{E}_{94}$ value of 0.46 for the 12 samples, which proves the excellent accuracy of the model. In transmittance mode, satisfying predictions are also obtained, even though slightly less accurate than in reflectance model with an average $\Delta \mathrm{E}_{94}$ value of 1.15. 


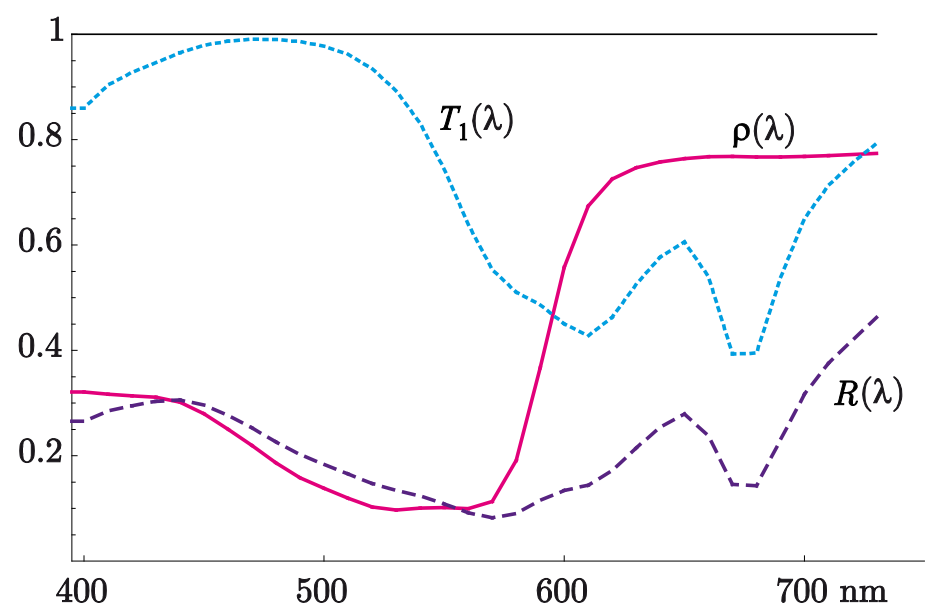

Figure 17. Spectral reflectance of (solid line:) a magenta printed paper and of (dashed line:) this paper covered by a blue acetate film of normal transmittance $T_{1}(\lambda)$.

A second application of the model was carried out from 125 printed CMY halftones printed by a Canon Pixma Pro9500 inkjet printer on Canon MP101 papers, and covered them by a standard transparent film for electrophotographic printing. Predictions were performed in reflectance mode and compared to the measured spectra. Good prediction accuracy is also proved by obtaining an average $\Delta \mathrm{E}_{94}$ value of 0.50 . This experiment is representative of the model's capacity to predict the change in spectral reflectance of prints when they are placed under glass or under protection film. This change in reflectance, and therefore of color, is not only due to the wavelength-dependent absorption of light by the protection film, but also on the direct reflection of light by the film and the multiple reflections of light between the film and the print.
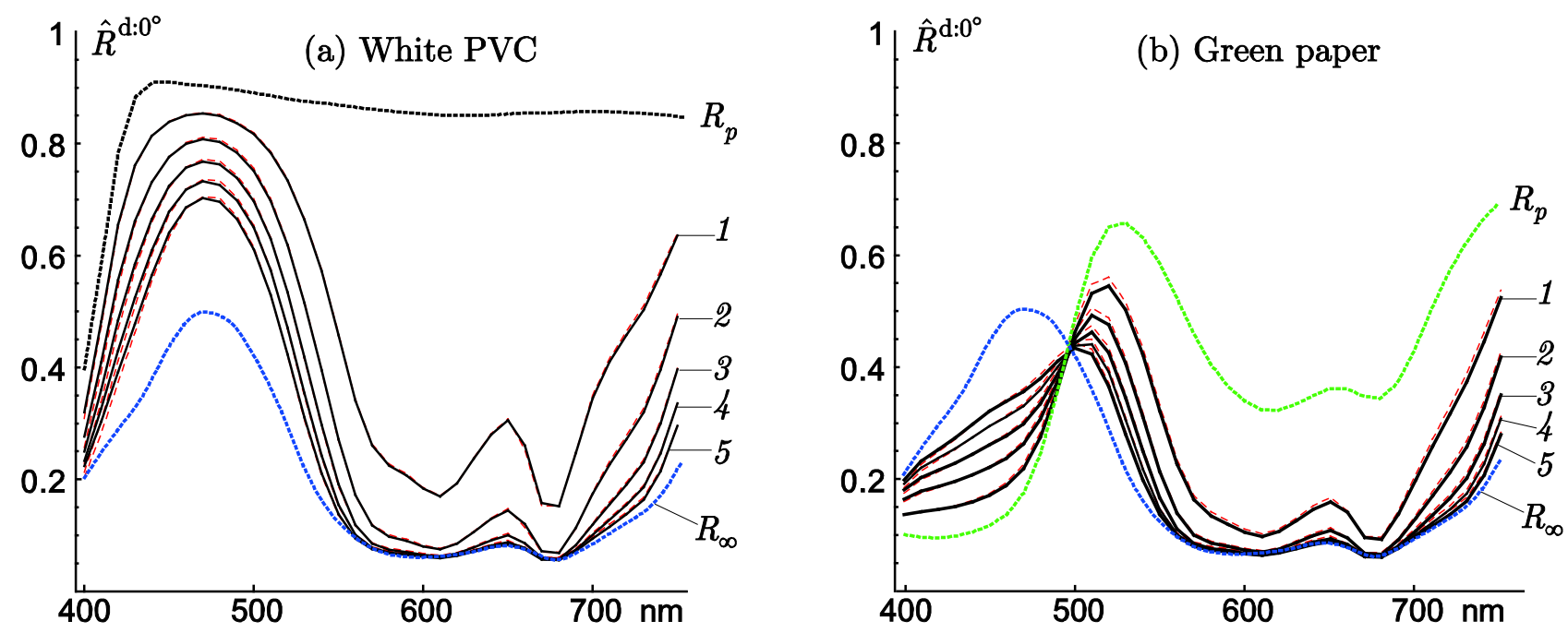
Figure 18. Measured (solid lines) and predicted (dashed lines) spectral reflectances of 1 to 5 acetate films on top of (a) white PVC and (b) green paper with respective reflectance $R_{p}$ plotted in dotted line. $R_{\infty}$ denotes the reflectance of an infinite stack of films.

A third application of the model was based on stacks of blue acetate films deposited on top of green glossy paper and white glossy PVC. One to five films were deposited in each case. The spectral reflectances measured with a d: $8^{\circ}$ geometry (or equivalently with a d: $0^{\circ}$ geometry) as well as the predicted ones are plotted in Figure 18. The $\Delta \mathrm{E}_{94}$ values computed between predicted and measured spectra are all below 0.25 unit in the case of the white PVC and 0.49 unit in the case of the green paper, thus showing the high accuracy of the model. In the case of the green paper, we observe similar phenomenon as for film in front of a specular background: the different spectra have same value at a given wavelength, presently $500 \mathrm{~nm}$. This invariance occurs precisely at the wavelength where the reflectance of the paper, $R_{p}$, coincides with the reflectance $R_{\infty}$ of the infinite stack of films. This is well-known in the case of oil paintings, particularly regarding the art-glaze technique where a white diffusing board is coated with several weakly pigmented, almost nonscattering layers $[1,2]$.

\section{MULTIFLUX MODELS}

The shortcomings of the two-flux model regarding collimated light and forward light scattering can be addressed by taking additional fluxes into account, corresponding to various angular distributions. This is especially needed when the medium is sufficiently scattering to not be considered as non-scattering, and not sufficiently scattering to be considered as strongly diffusing, i.e. Lambertian. We thus come back to the Kubelka-Munk formalism where the propagation of light in uniform layers is expressed in terms of absorption and scattering coefficients. Here, we present two classical configurations based on both collimated and diffuse fluxes: the four-flux model [22, 23], and the three-flux model [24].

\subsection{The four-flux model}

Let us consider an upward collimated flux, denoted $J_{\theta}$, and a downward collimated flux denoted $I_{\theta}$, where $\theta$ is the angle of incidence of the collimated beam. Looking at a slice of thickness $d x$, the upward oriented flux is attenuated by $K d x / 2 \cos \theta$ due to absorption and $S d x / \cos \theta$ due to scattering. The same process applies also to the downward oriented flux. For the collimated fluxes, there is no gain from any other flux. The variation of intensity is summarized in the following equation system: 


$$
\left\{\begin{array}{l}
\cos \theta \cdot \frac{d J_{\theta}}{d x}=-\frac{K}{2} J_{\theta}-S J_{\theta} \\
\cos \theta \cdot \frac{d I_{\theta}}{d x}=\frac{K}{2} I_{\theta}+S I_{\theta}
\end{array}\right.
$$

Applying the same matrix form as for the Kubelka-Munk model yields:

$$
\left(\begin{array}{c}
J_{\theta}(h) \\
I_{\theta}(h)
\end{array}\right)=\exp \left[\left(\begin{array}{cc}
-\frac{K}{2}-S & 0 \\
0 & \frac{K}{2}+S
\end{array}\right)\left(\frac{h-0}{\cos \theta}\right)\right] \cdot\left(\begin{array}{c}
J_{\theta}(0) \\
I_{\theta}(0)
\end{array}\right)
$$

Note that the amounts of light lost by scattering by $I_{\theta}$ and $J_{\theta}$ contributes to the diffuse fluxes $i$ and $j$. Combining equations (142) and (8) leads to

$$
\left(\begin{array}{c}
J_{\theta}(h) \\
I_{\theta}(h) \\
j(h) \\
i(h)
\end{array}\right)=\mathbf{M}_{4 K, S, h} \cdot\left(\begin{array}{c}
J_{\theta}(0) \\
I_{\theta}(0) \\
j(0) \\
i(0)
\end{array}\right)
$$

where $\mathbf{M}_{4 K, S, h}$ is the $4 \times 4$ matrix defined as follows:

$$
\mathbf{M}_{4 K, S, h}=\exp \left[\left(\begin{array}{cccc}
\frac{-1}{\cos \theta}\left(\frac{K}{2}+S\right) & 0 & 0 & 0 \\
0 & \frac{1}{\cos \theta}\left(\frac{K}{2}+S\right) & 0 & 0 \\
\frac{S}{2 \cos \theta} & \frac{S}{2 \cos \theta} & -(K+S) & S \\
-\frac{S}{2 \cos \theta} & -\frac{S}{2 \cos \theta} & -S & (K+S)
\end{array}\right)(h-0)\right]
$$

The same calculation methods apply to this $4 \times 4$ matrix denoted $\mathbf{M}_{4 K, S, h}$ as for the KubelkaMunk matrix $\mathbf{M}_{K, S, h}$ introduced in Section 2.2, equation (9). The matrix exponential, as defined by equation (7), can be calculated by diagonalization of $\mathbf{M}_{4, K, S}$, i.e. the matrix inside the exponential function in equation (144).

The Saunderson correction applies to $j(h)$ and $i(h)$ the same way as described in Section 2.7 for the Kubelka-Munk model. However, another transfer matrix must be defined for the collimated fluxes $I_{\theta}(0)$ and $J_{\theta}(0)$, similar to the one introduced in Section 4.2, equation (82). Assuming $n \sin \theta \leq 1$, the external collimated fluxes, denoted $I_{\psi}^{\prime}(0)$ and $J_{\psi}^{\prime}(0)$ where $\psi$ denotes the orientation of light in air, are related to the collimated fluxes in the medium, 
denoted $I_{\theta}(0)$ and $J_{\theta}(0)$, by the Fresnel angular reflectances using the transfer matrix $F_{12}(\psi)$ defined as in equation (72):

$$
\begin{aligned}
\left(\begin{array}{c}
J_{\psi}^{\prime}(0) \\
I_{\psi}^{\prime}(0)
\end{array}\right) & =\mathbf{F}_{\mathbf{1 2}}(\psi) \cdot\left(\begin{array}{c}
J_{\theta}(h) \\
I_{\theta}(h)
\end{array}\right) \\
& =\frac{1}{1-R_{01}(\psi)}\left(\begin{array}{cc}
1-2 R_{01}(\psi) & R_{01}(\psi) \\
-R_{01}(\psi) & 1
\end{array}\right) \cdot\left(\begin{array}{c}
J_{\theta}(h) \\
I_{\theta}(h)
\end{array}\right)
\end{aligned}
$$

Combining equations (145) and (44) yields a $4 \times 4$ Saunderson correction matrix:

$$
\mathbf{M}_{4 \text { Saunderson }}=\left(\begin{array}{cc}
\mathbf{F}_{\mathbf{0 1}}(\psi) & 0 \\
0 & \mathbf{f}_{\mathbf{0 1}}
\end{array}\right)=\left(\begin{array}{cccc}
\frac{1-2 R_{01}(\psi)}{1-R_{01}(\psi)} & \frac{R_{01}(\psi)}{1-R_{01}(\psi)} & 0 & 0 \\
\frac{-R_{01}(\psi)}{1-R_{01}(\psi)} & \frac{1}{1-R_{01}(\psi)} & 0 & 0 \\
0 & 0 & \frac{1-r_{01}\left(1+n^{2}\right)}{n^{2}\left(1-r_{01}\right)} & \frac{r_{01}}{1-r_{01}} \\
0 & 0 & \frac{\left(1-r_{01}\right)-n^{2}}{n^{2}\left(1-r_{01}\right)} & \frac{1}{1-r_{01}}
\end{array}\right)
$$

Combining equation (146) and equation (143) yields a new relation that relates the fluxes in the external medium with the fluxes at the bottom of the layer:

$$
\left(\begin{array}{c}
J_{\psi}^{\prime}(0) \\
I_{\psi}^{\prime}(0) \\
j^{\prime}(0) \\
i^{\prime}(0)
\end{array}\right)=\mathbf{M}_{4 \text { Saunderson }} \cdot \mathbf{M}_{4 K, S, h} \cdot\left(\begin{array}{c}
J_{\theta}(0) \\
I_{\theta}(0) \\
j(0) \\
i(0)
\end{array}\right)
$$

where $\psi$ and $\theta$ denote the orientations of light in air, and respectively in the medium.

\subsection{The three-flux model}

In most experimental set-ups a Lambertian reflector is used as background for the medium to be analyzed. As a consequence the incident collimated light beam is turned into a Lambertian light flux after the first reflection on the background: $J(0)=0$ and $j(0)=R_{g}(i(0)+I(0))$. The four-flux model can be simplified accordingly: 


$$
\left(\begin{array}{c}
I(h) \\
j(h) \\
i(h)
\end{array}\right)=\exp \left[\left(\begin{array}{ccc}
\frac{1}{\cos \theta}\left(\frac{K}{2}+S\right) & 0 & 0 \\
\frac{S}{2 \cos \theta} & -(K+S) & S \\
\frac{-S}{2 \cos \theta} & -S & K+S
\end{array}\right)(h-0)\right] \cdot\left(\begin{array}{c}
I(0) \\
j(0) \\
i(0)
\end{array}\right)
$$

For decades, the three-flux model was the most effective model to be implemented in color formulation packages. It offered a good compromise between precision and computation effort [11].

In the special case of a non-scattering material $(S=0)$ in optical contact with a substrate of reflectance $R_{g}$, equation (148) simplifies as follows:

$$
\begin{aligned}
\left(\begin{array}{c}
I(h) \\
j(h) \\
i(h)
\end{array}\right) & =\exp \left[\left(\begin{array}{ccc}
\frac{K}{2 \cos \theta} & 0 & 0 \\
0 & -K & 0 \\
0 & 0 & K
\end{array}\right)(h-0)\right] \cdot\left(\begin{array}{c}
I(0) \\
j(0) \\
i(0)
\end{array}\right) \\
& =\left(\begin{array}{ccc}
e^{\frac{K h}{2 \cos \theta}} & 0 & 0 \\
0 & e^{-K h} & 0 \\
0 & 0 & e^{K h}
\end{array}\right) \cdot\left(\begin{array}{c}
I(0) \\
R_{g} \cdot(i(0)+I(0)) \\
i(0)
\end{array}\right)
\end{aligned}
$$

\section{CONCLUSIONS}

The main interest of the two-flux models, i.e. the Kubelka-Munk model and Kubelka's layering model, lies in the analytical expressions they provide for reflectance and the transmittance of layers and stacks of layers. This is probably the reason of their amazing success. In these analytical expressions, the parameters attached to the layers can be directly or indirectly related to reflectances and transmittances measured using a spectrophotometer, and those attached to the interfaces can be computed from the Fresnel formulas when the optical indices are known. Thanks to the extensions presented here based on transfer matrices, the Kubelka-Munk model, Kubelka's model and Saunderson's correction can be easily transposed to more complex configurations, for example the case of many layers with distinct optical indices. They thus enable the prediction of the visual rendering of many common colored surfaces such as inked papers, paintings, photographs, protection films... Fairly good prediction accuracy can be achieved provided the implicit assumptions of the two-flux approach are well satisfied. The Kubelka-Munk model assumes homogeneous, significantly 
scattering layers and Lambertian illumination. In Kubelka's model, layers are not necessarily homogenous: their scattering and absorption coefficients can vary as a function of the depth, and in a stack, they can have distinct optical indices. The model applies with stacks of strongly scattering layers illuminated with Lambertian flux, and with stacks of nonscattering layers illuminated with collimated flux. By extension, the model also applies to stacks of nonscattering layers illuminated with diffuse flux by considering this diffuse flux as a collection of collimated rays: the angular reflectances and transmittances yielded by the model are summed up over the range of orientations of the rays. Thanks to this, stacks of strongly scattering and nonscattering layers can also be addressed provided the geometry of illumination for each layer is specified.

When these assumptions are not satisfied, the two-flux approach may fail and more complex models must be used. The three-flux and four-flux models extending the Kubelka-Munk model, presented in Section 6, apply for example in the case of scattering layers illuminated by collimated light. There exist other approaches, for example a numerical solving of the radiative transfer equation (see [HDI062], Section 6, as well as reference [24] for the relationship between the radiative transfer theory and the three- and four-flux approximations), but they generally need modeling the optical properties of the media at small scale as well as more important computational effort, while no analytical expression can be expected. This is the reason why in most applications the two-flux approach is tested at first and, when it is not accurate enough, simple extensions are often developed hoping to reach the expected prediction accuracy.

\section{APPENDIX - ALTERNATIVE DEFINITION OF TRANSFER MATRICES}

The transfer matrix of a flat interface as defined in Section 4.2 by equation (82) becomes indefinite when the orientation of light exceeds the critical angle because the reflectance $R_{j k}$ is 1 , therefore the transmittance $T_{j k}$ is 0 . In order to prevent computational problems, we recommend using an alternative general definition for the transfer matrices where the downward transmittance is incorporated into the matrix in a third row and third column:

$$
\mathbf{M}=\left(\begin{array}{ccc}
\tau \tau^{\prime}-\rho \rho^{\prime} & \rho & 0 \\
-\rho^{\prime} & 1 & 0 \\
0 & 0 & \tau
\end{array}\right)
$$


where $\rho, \rho$ ', $\tau, \tau$ resp etiv dy deno e the $u \mathbb{p} \notin$ reflectance, the back reflectance, the downward reflectance and the upward transmittance.

These $3 \times 3$ matrices can be multiplied in the same way as the $2 \times 2$ transfer matrices without any chance of division by zero. Reflectances and transmittances are deduced from the entries $m_{i j}(i, j=1,2,3)$ of the $3 \times 3$ matrix in the following way:

$$
\begin{aligned}
& \rho=m_{12} / m_{22} \\
& \tau=m_{33} / m_{22} \\
& \rho^{\prime}=-m_{12} / m_{22} \\
& \tau^{\prime}=\left[\operatorname{det}(\mathbf{M}) / m_{22}\right] / m_{33}^{2}
\end{aligned}
$$

Let us examine what happens in the matrix product of equation (90) when a total reflection occurs at the middle interface. The transfer matrix of the middle interface, $\mathbf{F}_{12}\left(\theta_{1}\right)$, is given by (82) with the Fresnel reflectance $R_{12}\left(\theta_{1}\right)$ equal to 1 . After conversion into the $3 \times 3$ matrix format, we have

$$
\mathbf{F}_{12}\left(\theta_{1}\right)=\left(\begin{array}{ccc}
-1 & 1 & 0 \\
-1 & 1 & 0 \\
0 & 0 & 0
\end{array}\right)
$$

With this matrix $\mathbf{F}_{12}\left(\theta_{1}\right)$, the product of matrices in equation (90) provides the following front reflectance for the multilayer, which contains no terms relative to the second layer or the back interface:

$$
R_{0123}\left(\theta_{0}\right)=R_{01}\left(\theta_{1}\right)+\frac{T_{10}\left(\theta_{1}\right) T_{01}\left(\theta_{0}\right) t_{1}^{2 / \cos \theta_{1}}}{1-R_{10}\left(\theta_{1}\right) t_{1}^{2 / \cos \theta_{1}}}
$$

The fact that no light enters the second layer is therefore automatically taken into account by the model. The downward transmittance is zero. The back reflectance and the upward transmittance, in this case, make no sense anymore.

\section{REFERENCES}

1. Elias, M, Simonot, L. (2006) "Separation between the different fluxes scattered by art glazes: Explanation of the special color saturation", Applied Optics 45, 3163-3172.

2. Simonot, L, Elias, M, Charron, E. (2004) "Special visual effect of art-glazes explained by the radiative transfer equation" Applied Optics 43, 2580-2587.

3. Hébert, M, Hersch, RD, Becker, JM, (2007) "Compositional reflectance and transmittance model for multilayer specimens," J. Opt. Soc. Am. A 24, 2628-2644.

4. Kubelka, P, Munk, F. (1931) "Ein Beitrag zur Optik der Farbanstriche" Zeitschrift für technische Physik 12, 593-601. 
5. Kubelka, P. (1948) "New contributions to the optics of intensely light-scattering material, part I" J. Opt. Soc. Am. 38, 448-457.

6. Kubelka, P. (1954) "New contributions to the optics of intensely light-scattering materials, part II: Non homogeneous layers" J. Opt. Soc. Am. 44, 330-335.

7. ASTM D1003 (2011) Standard Test Method for Haze and Luminous Transmittance of Transparent Plastics.

8. Horn R. A., Johnson C. R., Topics in Matrix Analysis, Cambridge University Press, 1994, pp. 407-449.

9. Wyszecki G., Stiles W.S., Color Science: Concepts and Methods, Quantitative Data and Formulae, Second Edition, John Wiley \& Sons, New York, 1982, p.785.

10. Yang, L, Hersch, RD, (2008) Kubelka-Munk Model for imperfectly diffuse light distribution in paper, Journal of the Imaging Science and Technology 52, 030201-7.

11. Pauli H., Eitel D., "Comparison of Different Theoretical Models of Multiple Scattering for Pigmented Media", Colour 73, 1973, pp. 423-426.

12. Heavens, OS. (1960) "Optical properties of thin films" Rep. Prog. Phys. 23, p. 12.

13. Mitsas, CL, Siapkas, DI. (1995) "Generalized matrix method for analysis of coherent and incoherent reflectance and transmittance of multilayer structures with rough surfaces, interfaces, and finite substrates," Appl. Opt. 34, 1678-1683.

14. Katsidis, CC, Siapkas, DI. (2002) "General Transfer-Matrix Method for Optical Multilayer Systems with Coherent, Partially Coherent, and Incoherent Interference," Appl. Opt. 41, 39783987.

15. Hébert, M, Hersch, RD (2006) "A reflectance and transmittance model for recto-verso halftone prints" J. Opt. Soc. Am. A 22, 1952-1967.

16. Strang, G. (1986) Applied Mathematics, Cambridge, MA, MIT Press.

17. Hébert, M, Hersch, RD, Simonot, L. (2008) "Spectral prediction model for piles of nonscattering films" J. Opt. Soc. Am. A 25, 2066-2077.

18. Hébert, M, Machizaud, J. (2012) "Spectral reflectance and transmittance of stacks of nonscattering films printed with halftone colors" J. Opt. Soc. Am. A 29, 2498-2508

19. Ostromoukhov, V, Hersch, RD. (1999) "Stochastic clustered-dot dithering" J. Electronic Imaging 8, 439.

20. Williams, FC, Clapper, FR. (1953) "Multiple Internal Reflections in Photographic Color Prints" J. Opt. Soc. Am. 43, 595-597.

21. Shore, JD, Spoonhower, JP. (2001) "Reflection Density in Photographic Color Prints: Generalizations of the Williams-Clapper Transform" J. of Imaging Science and Technology 45, 484-488.

22. Maheu, B, Letouzan, JN, Gouesbet, G. (1984) Four-flux models to solve the scattering transfer equation in terms of Lorentz-Mie parameters. Applied Optics 23, 3353-3362.

23. Maheu, B, Gouesbet, G. (1986) Four-flux models to solve the scattering transfer equation: special cases. Applied Optics 25, 1122-1128.

24. Klein, GA. (2010) Industrial Color Physics, Springer Verlag, Berlin. 